\title{
The Effect of Galaxy Interactions on Molecular Gas Properties
}

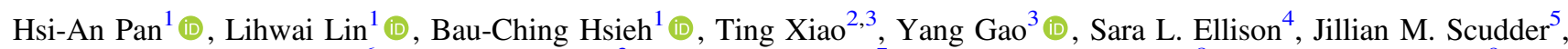
Jorge Barrera-Ballesteros ${ }^{6}$ (1) , Fangting Yuan ${ }^{3}$, Amélie Saintonge ${ }^{7}$ (1) Christine D. Wilson ${ }^{8}$ (1), Ho Seong Hwang ${ }^{9}$ (1),

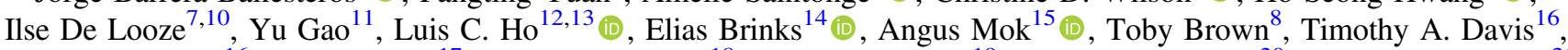
Thomas G. Williams $^{16}$, Aeree Chung ${ }^{17}$ (iD), Harriet Parsons ${ }^{18}$ (1) , Martin Bureau ${ }^{19}$ (i) , Mark T. Sargent ${ }^{20}$ (i), Eun Jung Chung ${ }^{21}$ (D), Eunbin Kim ${ }^{21,22}$, Tie Liu ${ }^{18,21,23}$ (1), Michał J. Michałowski ${ }^{24}$, and Tomoka Tosaki ${ }^{25}$

${ }^{1}$ Institute of Astronomy and Astrophysics, Academia Sinica, AS/NTU Astronomy-Mathematics Building, No. 1, Section 4, Roosevelt Rd., Taipei 10617, Taiwan

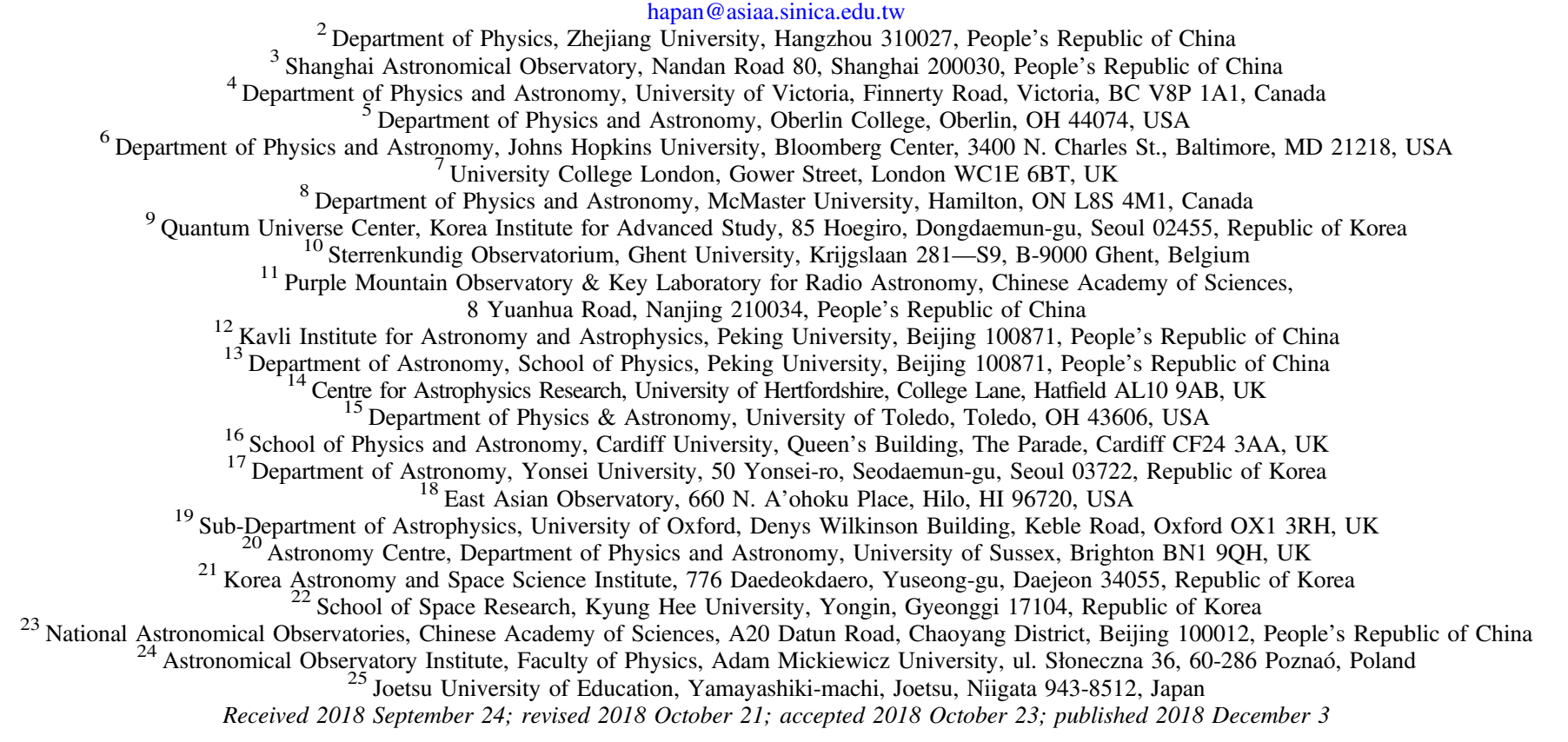
hapan@asiaa.sinica.edu.tw

\begin{abstract}
Galaxy interactions are often accompanied by an enhanced star formation rate (SFR). Since molecular gas is essential for star formation, it is vital to establish whether and by how much galaxy interactions affect the molecular gas properties. We investigate the effect of interactions on global molecular gas properties by studying a sample of 58 galaxies in pairs and 154 control galaxies. Molecular gas properties are determined from observations with the JCMT, PMO, and CSO telescopes and supplemented with data from the xCOLD GASS and JINGLE surveys at ${ }^{12} \mathrm{CO}(1-0)$ and ${ }^{12} \mathrm{CO}(2-1)$. The SFR, gas mass $\left(M_{\mathrm{H}_{2}}\right)$, and gas fraction $\left(f_{\text {gas }}\right)$ are all enhanced in galaxies in pairs by $\sim 2.5$ times compared to the controls matched in redshift, mass, and effective radius, while the enhancement of star formation efficiency (SFE $\equiv \mathrm{SFR} / M_{\mathrm{H}_{2}}$ ) is less than a factor of 2 . We also find that the enhancements in SFR, $M_{\mathrm{H}_{2}}$ and $f_{\mathrm{gas}}$, increase with decreasing pair separation and are larger in systems with smaller stellar mass ratio. Conversely, the SFE is only enhanced in close pairs (separation $<20 \mathrm{kpc}$ ) and equal-mass systems; therefore, most galaxies in pairs lie in the same parameter space on the SFR- $M_{\mathrm{H}_{2}}$ plane as controls. This is the first time that the dependence of molecular gas properties on merger configurations is probed statistically with a relatively large sample and a carefully selected control sample for individual galaxies. We conclude that galaxy interactions do modify the molecular gas properties, although the strength of the effect is dependent on merger configuration.
\end{abstract}

Key words: galaxies: interactions - galaxies: ISM - ISM: molecules - galaxies: star formation

\section{Introduction}

It has been well established that galaxy interactions can trigger bursts of star formation. Interaction-triggered star formation was first observed by Larson \& Tinsley (1978), who found that interacting galaxies have large dispersion in $U-B / B-V$ colors due to the short-duration starbursts. Since then, many observations have confirmed this finding (e.g., Darg et al. 2010a; Scudder et al. 2012; Knapen et al. 2015). Observationally, the strongest starbursts (e.g., ultraluminous infrared galaxies (ULIRGs)) are predominantly merging systems (Sanders et al. 1988; Borne et al. 1999), which supports the idea that galaxy interactions are efficient in converting gas to stars. Simulations also show that external perturbations can trigger star formation by the gas inflow induced as a result of tidal forces (e.g., Barnes \& Hernquist 1996; Di Matteo et al. 2007; Moreno et al. 2015).

However, an enhanced star formation rate (SFR) is not ubiquitous in interacting galaxies. The average level of the SFR enhancement of galaxy pairs, as measured in both observations and simulations, is moderate, typically below a factor of a few 
(Di Matteo et al. 2007, 2008; Lin et al. 2007; Martig \& Bournaud 2008; Hwang et al. 2011; Wong et al. 2011; Scudder et al. 2012; Xu et al. 2012; Ellison et al. 2013; Knapen et al. 2015). Star formation enhancement depends on parameters such as separation between galaxies in pairs (Lambas et al. 2003; Ellison et al. 2008; Park \& Choi 2009; Hwang et al. 2010; Scudder et al. 2012; Patton et al. 2013; Davies et al. 2015), the properties of the progenitor galaxies (Mihos \& Hernquist 1996; Cox et al. 2008; Xu et al. 2010; Scudder et al. 2012; Davies et al. 2015), merging geometry (Springel \& Hernquist 2005; Di Matteo et al. 2007; Moreno et al. 2015; Sparre \& Springel 2016), and gas (H I) fraction (Scudder et al. 2015).

Given that SFR depends on the molecular gas reservoir (Kennicutt 1998a), one would expect that the amount or physical properties of the molecular gas change while a galaxy undergoes an interaction with another galaxy (e.g., Moreno et al. 2018). Two possibilities for enhanced star formation in galaxy pairs are most commonly proposed: (1) an enrichment of the molecular gas reservoir, which fuels star formation (e.g., Combes et al. 1994; Casasola et al. 2004), and (2) an increase in the efficiency of converting gas into stars (e.g., Solomon \& Sage 1988; Sofue et al. 1993; Michiyama et al. 2016). Both scenarios have observationally testable predictions - the former predicts higher molecular gas mass $\left(M_{\mathrm{H}_{2}}\right)$, or, more precisely, a higher molecular gas mass fraction with respect to the total (gas and stars) mass $\left(f_{\text {gas }}\right)$, while the latter predicts a higher star formation efficiency (SFE) of molecular gas.

However, observations of $\mathrm{H}_{2}$ in interacting galaxies have yet to give a clear picture of whether it is the total gas reservoir or the SFE that drives the enhanced SFR in galaxy pairs. Solomon \& Sage (1988) observed ${ }^{12} \mathrm{CO}(1-0)$ in 93 far-infrared-bright pairs and classified them into five types according to the degree of interaction. They found that there is no significant difference in CO luminosity (given that $L_{\mathrm{CO}} \propto M_{\mathrm{H}_{2}}$ ) between their pair types and isolated galaxies. On the other hand, strong interactions give rise to an increase in $L_{\mathrm{FIR}} / L_{\mathrm{CO}}$ ratio $(\propto \mathrm{SFE})$. Sofue et al. (1993) also found an elevated SFE in their 54 interacting galaxies taken from the Arp Atlas. More recently, Michiyama et al. (2016) revealed an increasing SFE from isolated to interacting galaxies and from early-stage to late-stage interactions using a sample of 60 interacting and 28 isolated galaxies. ${ }^{26}$ Yet several studies have arrived at the opposite conclusion. For example, Combes et al. (1994) observed ${ }^{12} \mathrm{CO}(1-0)$ in 51 interacting galaxies and found that the total molecular content increases with decreasing projected separation of the pairs, while SFE does not. Accordingly, they concluded that the total molecular content plays a more significant role in triggering star formation than SFE. A similar result is also reached by Casasola et al. (2004) using several hundred interacting galaxies and 2000 normal galaxies compiled from the literature.

Although the above results indicate that galaxy interactions may affect the molecular gas properties, the above analyses have several shortcomings that might contribute to their conflicting results. For example, the global properties of the control galaxies to be compared with pairs should be carefully controlled. The majority of previous studies compare the properties of interacting and isolated galaxies directly, where the latter may not always be the perfect reference in terms of the distributions of their redshift, stellar mass $\left(M_{*}\right)$, and other galaxy properties.

\footnotetext{
${ }^{26}$ But note that they use ${ }^{12} \mathrm{CO}(3-2)$ as a molecular gas tracer, which may not trace total gas content due to the high critical density of ${ }^{12} \mathrm{CO}(3-2)$ (a few times $10^{3} \mathrm{~cm}^{-3}$ ).
}

Another important factor is the choice of CO-to- $\mathrm{H}_{2}$ conversion factor $\left(\alpha_{\mathrm{CO}}\right)$ between the measured $L_{\mathrm{CO}}$ and $M_{\mathrm{H}_{2}}$. The validity of the widely adopted Galactic $\alpha_{\mathrm{CO}}$ is often questioned (see the review by Bolatto et al. 2013). Empirically, $\alpha_{\mathrm{CO}}$ increases with decreasing gas-phase metallicity $12+\log (\mathrm{O} / \mathrm{H})$ due to the decreasing abundance of CO relative to $\mathrm{H}_{2}$ (Arimoto et al. 1996; Leroy et al. 2011; Narayanan et al. 2012). Since gas-phase metallicity is known to correlate with the $M_{*}, M_{\mathrm{H}_{2}}$, and SFR of a galaxy (Tremonti et al. 2004; Mannucci et al. 2010; Bothwell et al. 2016), a physically motivated $\alpha_{\mathrm{CO}}$ is essential for the study of molecular gas in galaxies.

Recently, Violino et al. (2018) took a step toward addressing these improvements by considering a control sample with properties matched to the galaxy pairs and using a physically motivated $\alpha_{\mathrm{CO}}$. They found that galaxy pairs have higher SFE and $f_{\text {gas }}$ compared to the control sample. However, the investigation of the relation between merger configuration and gas properties is limited by the small sample size (11 galaxies in pairs) in Violino et al. (2018). It remains untested about how the change in gas properties correlates with the details of galaxy interaction properties, such as pair separation and mass ratio.

In this paper, we study molecular gas properties, which are calculated using a physically motivated value of $\alpha_{\mathrm{CO}}$, toward a sample of 58 galaxies in pairs. We compare their star formation and molecular gas properties with a sample of carefully matched control galaxies. The sample uniquely covers major and minor mergers (from equal-mass merger to a ratio of $\sim 100$ ), widely separated pairs and close pairs, and primary (higher $M_{*}$ ) and secondary (lower $M_{*}$ ) galaxies in a pair. This is the first time that the dependence of molecular gas properties on merger properties is probed statistically with a relatively large sample and carefully selected control sample for individual galaxies in pairs.

This paper is organized as follows. In Section 2, we describe our pair identification and data used in the analysis. In Sections 3.1 and 3.2, we first compare the SFR and molecular gas properties of the galaxies in pairs and the control sample by comparing the medians of the two samples. Next, in Sections 3.3 and 3.4, we explore the dependence of SFR and gas properties on merger properties, including projected separation and the stellar mass ratio of the two galaxies in a pair. In Section 4, we discuss the potential driver of star formation in galaxies in pairs and the locus of our galaxies in the $\mathrm{SFR}-M_{\mathrm{H}_{2}}$ relation. The main results are summarized in Section 5.

Throughout this paper, we assume $\Omega_{\mathrm{m}}=0.3, \Omega_{\Lambda}=0.7$, $H_{0}=70 \mathrm{~km} \mathrm{~s}^{-1} \mathrm{Mpc}^{-1}$, and a Kroupa initial mass function (IMF) of stars (Kroupa 2001).

\section{Data \\ 2.1. Molecular Gas Observations of Galaxies in Pairs}

The pair samples in this work are either obtained by our group or compiled from several surveys, summarized in Table 1. The final sample consists of 58 galaxies in pairs and 154 isolated galaxies from which the control galaxies are drawn. The galaxies in pairs we refer to here are galaxies with a spectroscopic or morphological companion. In most of the cases discussed in this paper, the molecular gas observations were made toward one of the galaxies in a pair, except a few close pairs. Emission from the companion might be detected 
Table 1

Summary of the Observations

\begin{tabular}{lccccc}
\hline \hline & & Galaxies in Pairs & Pool of Controls \\
\hline Project & PI programs & JINGLE & JINGLE Pilot & xCOLD GASS \\
Number & 21 & 5 & $2+2+1$ & 27 & xCOLD GASS \\
Telescope & JCMT & JCMT & JCMT $/$ PMO $/ \mathrm{CSO}$ & 154 \\
Tracer & ${ }^{12} \mathrm{CO}(2-1)$ & ${ }^{12} \mathrm{CO}(2-1)$ & ${ }^{12} \mathrm{CO}(2-1) /(1-0) /(2-1)$ & ${ }^{12} \mathrm{CO}(1-0)$ & IRAM \\
Beam size & $22^{\prime \prime}$ & $22^{\prime \prime}$ & $22^{\prime \prime} / 52^{\prime \prime} / 30^{\prime \prime}$ & $22^{\prime \prime}$ & ${ }^{12} \mathrm{CO}(1-0)$ \\
\hline
\end{tabular}

for the pairs with the smallest projected separations. The potential effect of this contamination will be discussed in Section 4.1. Details of the sample selection, observations, and data reduction are described in this section.

\subsubsection{Pair Sample: JCMT Observations (PI Programs)}

The molecular gas observations of about half of the galaxies in pairs were obtained through our two PI programs on the James Clerk Maxwell Telescope (JCMT; project codes: M17AP060 and M17BP053; PI: H.-A. Pan).

The pair sample was selected from the 2779 galaxies in the fifth product launch (MPL-5, corresponding to SDSS DR13) of Mapping Nearby Galaxies at APO (MaNGA). MaNGA is part of the fourth generation of the Sloan Digital Sky Survey (SDSS-IV; Gunn et al. 2006; Blanton et al. 2017) and aims to obtain spatially resolved spectroscopy of 10,000 galaxies with a median redshift of $\sim 0.03$ by 2020 . MaNGA has a wavelength coverage of $3600-10300 \AA$, with a spectral resolution varying from $R \sim 1400$ at $4000 \AA$ to $R \sim 2600$ around $9000 \AA$. Further details of the science goals, as well as sample selection, can be found in Bundy et al. (2015) and Wake et al. (2017). While this work focuses on the globally integrated star formation and molecular gas properties, the existence of MaNGA data will be beneficial in advancing the analysis of the spatially resolved properties in the future.

We first identify galaxies in pairs in these 2779 MaNGA galaxies. The galaxies in pairs are defined as those systems with projected separation $\left(r_{p}\right)<50 \mathrm{kpc} \mathrm{h}^{-1}$ (around $71.4 \mathrm{kpc}$ with $h=0.7)$ and line-of-sight velocity difference $(\Delta V)<$ $500 \mathrm{~km} \mathrm{~s}^{-1}$ (e.g., Patton et al. 2002; Lin et al. 2004). It has been noticed that the SFR enhancement can extend out to $150 \mathrm{kpc}$ (Patton et al. 2013), although the enhancement for $r_{p}>$ $100 \mathrm{kpc}$ is almost negligible. Moreover, even with spectroscopic redshifts, interlopers may still exist and become a more significant effect with larger separation. For these reasons, the criterion of $50 \mathrm{kpc} \mathrm{h}^{-1}$ seems to be a reasonable choice. These criteria identified 662 galaxies in pairs. However, if the two merger components are too close (normally late-stage mergers) to be deblended by SDSS or do not have two separate spectroscopic redshift measurements, they will not be identified as galaxies in pairs. To pick up those late-stage systems, we use the " $P$-merger" parameter (weighted-merger-vote fraction) from Galaxy Zoo (Darg et al. 2010a, 2010b). The $P$-merger parameter quantifies the probability that an object is a merger based on visual inspection of large numbers of objects by human classifiers. It ranges from 0 , an object looks nothing like a merger, to 1 , an object looks unmistakably so. The criteria of $P$-merger $>0.4$ suggested by Darg et al. (2010a, 2010b) is applied to select galaxies in pairs. The number of galaxies in pairs in the MaNGA sample increases to 736 by adding the Galaxy Zoo criterion.
The required observing time for JCMT ${ }^{12} \mathrm{CO}(2-1)$ observations for each of the identified galaxies in pairs is estimated in the following way. We first calculate the expected $M_{\mathrm{H}_{2}}$ from the Wide-field Infrared Survey Explorer $12 \mu \mathrm{m}$ luminosity $\left(L_{12} \mu \mathrm{m}\right)$ using the $L_{12} \mu \mathrm{m}-M_{\mathrm{H}_{2}}$ relation proposed by Jiang et al. (2015). Since $12 \mu \mathrm{m}$ emission is a good tracer of star formation (Donoso et al. 2012; Lee et al. 2013), the relation is essentially the same as the Kennicutt-Schmidt relation. The ${ }^{12} \mathrm{CO}(1-0)$ luminosity is calculated from $M_{\mathrm{H}_{2}}$ using the Milky Way value for $\alpha_{\mathrm{CO}}$ of $4.3 M_{\odot}\left(\mathrm{K} \mathrm{km} \mathrm{s}^{-1} \mathrm{pc}^{2}\right)^{-1}$ (Bolatto et al. 2013). Subsequently, the luminosity and flux of ${ }^{12} \mathrm{CO}(2-1)$ is derived assuming a ${ }^{12} \mathrm{CO}(2-1)$ to ${ }^{12} \mathrm{CO}(1-0)$ line intensity ratio $\left(R_{21}\right)$ of 0.6 . The adopted $R_{21}$ here is a conservative choice (lower limit) for time estimation, as $R_{21}$ is found to be $0.6-1.0$ in nearby galaxies (e.g., Braine \& Combes 1992; Leroy et al. 2009). Although we made conservative assumptions for $R_{21}$ and $\alpha_{\mathrm{CO}}$ for the purpose of estimating the exposure time, later we will use a different $R_{21}$ that is chosen based on gas properties; moreover, we will present a physically motivated $\alpha_{\mathrm{CO}}$ prescription that computes the value on a galaxy-by-galaxy basis in Section 3.1 and explore these assumptions in Section 4.4. Finally, we estimate the required time for a $4 \sigma$ detection with a velocity resolution of $30 \mathrm{~km} \mathrm{~s}^{-1}$ for each identified galaxy in pairs and propose to observe 41 galaxies that have a suitable declination range and require on-source time of $<250$ minutes each. Any possible bias introduced by this latter choice will be discussed in Section 4.1.

The ${ }^{12} \mathrm{CO}(2-1)(230.538 \mathrm{GHz})$ observations of the 41 galaxies in pairs were obtained by JCMT using the RxA3m receiver (but only 21 galaxies were used for the analysis after a further control on galaxy properties; we will show the criteria in the next paragraph). The redshift range of these galaxies in pairs is $0.02-0.06$. The beam size of the telescope is $22^{\prime \prime}$ at $230 \mathrm{GHz}$. The observations were conducted during several periods from 2016 November to 2018 January. The sky opacity at $225 \mathrm{GHz}$ was reported by the JCMT's Water Vapour Monitor (WVM), an in-cabin line-of-sight radiometer assessing the $183 \mathrm{GHz}$ water line (reported at $225 \mathrm{GHz}$ for historic reasons). The typical sky opacity was $0.08-0.20$. The typical system temperatures were between 200 and $400 \mathrm{~K}$. The onsource time for individual galaxies ranged from 40 minutes to $4 \mathrm{hr}$. The total on-source time for the two PI programs was $\sim 90 \mathrm{hr}$. The data reduction was done using the Starlink software (Currie et al. 2014). Individual exposures ( $\sim 20-40$ minutes, including calibration) of a given galaxy were calibrated separately and then coadded. The spectrum was binned to a velocity resolution of $30 \mathrm{~km} \mathrm{~s}^{-1}$. A linear baseline was subtracted from the spectrum using line-free channels. For a few galaxies for which the baseline is structured, a second- or third-order polynomial was used to subtract the baseline. Spectra were converted from antenna temperature in K to Jy by applying a factor of $15.6 / \eta_{a}$, where the aperture efficiency $\eta_{a}$ is 0.55 . The integrated CO luminosity $L_{\mathrm{CO}}$ is computed following 
Solomon et al. (1997),

$$
\frac{L_{\mathrm{CO}}}{\left(\mathrm{K} \mathrm{km} \mathrm{s}^{-1} \mathrm{pc}^{2}\right)}=3.27 \times 10^{7} S_{\mathrm{CO}} \nu_{\mathrm{CO}}^{-2} D_{L}^{2}(1+z)^{-3} .
$$

In this expression, $S_{\mathrm{CO}}$ is the line flux in units of $\mathrm{Jy} \mathrm{km} \mathrm{s}^{-1}, \nu_{\mathrm{CO}}$ is the observed frequency in $\mathrm{GHz}$, and $D_{L}$ represents the luminosity distance in Mpc. For ${ }^{12} \mathrm{CO}(2-1)$ observations, a ${ }^{12} \mathrm{CO}(2-1)$-to- ${ }^{12} \mathrm{CO}(1-0)$ intensity ratio of 0.8 is assumed when calculating $L_{\mathrm{CO}}$ (Leroy et al. 2009). If the gas is optically thick, a ratio of 0.8 corresponds to an excitation temperature of $\sim 10 \mathrm{~K}$. A detection (i.e., signal-to-noise ratio $(\mathrm{S} / \mathrm{N})>3$ ) rate of $\sim 90 \%$ is achieved, implying that the approach we have taken to calculate the required sensitivity is valid.

Although 41 galaxies in pairs were obtained from the PI programs, only 21 galaxies are used for the analysis in this paper. Since the control sample used in this work (Section 2.2) has a stellar mass cut of $\log \left(M_{*} / M_{\odot}\right)=9$ (Saintonge et al. 2017), we remove galaxies that have $M_{*}$ less than this value. Moreover, only spiral galaxies are used, because early-type galaxies potentially have lower SFR, $M_{\mathrm{H}_{2}}$, and $f_{\text {gas }}$, which may be irrelevant to the existence of interaction or not. The Galaxy Zoo property, a debiased probability of being a spiral galaxy "P-CS," is used to identify galaxy morphology. We select galaxies that have $\mathrm{P}-\mathrm{CS} \geqslant 0.6$. All of the 21 galaxies in pairs have a solid detection by JCMT. Of these, 20 are selected based on pair separation, and one is based on the Galaxy Zoo morphology.

\subsubsection{Pair Sample: JINGLE}

The JCMT dust and gas In Nearby Galaxies Legacy Exploration (JINGLE) is an ongoing JCMT Large Program (Saintonge et al. 2018). JINGLE is designed to systematically study the cold interstellar medium of galaxies in the local universe. The survey observed an $850 \mu \mathrm{m}$ dust continuum with SCUBA-2 for a sample of $193 \mathrm{Herschel}$-selected galaxies with $\log \left(M_{*} / M_{\odot}\right)>9$ and integrated ${ }^{12} \mathrm{CO}(2-1)$ line fluxes with RxA3m for a subset of 97 of these galaxies. Of the 97 galaxies, 63 are within the footprint of MaNGA.

We briefly summarize the sample selection of JINGLE here. The JINGLE parent sample consists of $\sim 2800$ galaxies with $\log \left(M_{*} / M_{\odot}\right)>9$ and $0.01<z<0.05$ within the North Galactic Pole (NGP) region and three of the equatorial Galaxy And Mass Assembly (GAMA) fields (GAMA09, GAMA12, and GAMA15). The sample is narrowed down to $\sim 280$ galaxies with a $>3 \sigma$ detection at both 250 and $350 \mu \mathrm{m}$ in the Herschel ATLAS survey that are predicted to be detectable with SCUBA-2 in less than $2 \mathrm{hr}$ of integration. Then, 193 galaxies are selected in order to have a uniform stellar mass distribution at $\log \left(M_{*} / M_{\odot}\right)>9$. A subsample of 97 galaxies predicted to be detectable in an on-source time of 345 minutes are selected to obtain integrated ${ }^{12} \mathrm{CO}(2-1)$ line fluxes. Two methods are used to estimate the flux and integration time for ${ }^{12} \mathrm{CO}(2-1)$ observations. The first method is the same as that used for the PI programs in Section 2.1.1. The second approach is based on the 2-Star Formation Mode formal flux prediction of Sargent et al. (2014), in which the ${ }^{12} \mathrm{CO}(1-0)$ line flux is related to the galaxy's position in the SFR $-M_{*}$ plane. The predicted fluxes from the two methods agree well with each other. We note that JINGLE adopts an $R_{21}$ of 0.7 for the required sensitivity and observing time estimation. More details on the JINGLE design, as well as the sample selection and science goals, are given by Saintonge et al. (2018; overview of the survey), M. W. L. Smith et al. (2018, in preparation; details of dust observations), and T. Xiao et al. (2018, in preparation; details of molecular gas observations).

We apply the same criteria to select galaxies in pairs as described in Section 2.1.1 $\left(r_{p}<50 \mathrm{kpch}^{-1}\right.$ and $\Delta V<$ $500 \mathrm{~km} \mathrm{~s}^{-1}$, or $P$-merger $>0.4$ and P-CS $>0.6$ ) to the 45 JINGLE galaxies for which CO data were obtained before 2017 August. ${ }^{2 \gamma} \mathrm{A}$ total of five galaxies in pairs are identified in this way, all of them through the $r_{p}$ and $\Delta V$ criteria. The data reduction is carried out in the same way as our PI programs, described in Section 2.1.1.

\subsubsection{Pair Sample: JINGLE Pilot}

The JINGLE Pilot program (Y. Gao et al. 2018, in preparation) is a series of ${ }^{12} \mathrm{CO}(2-1)$ and (1-0) observations of MaNGA galaxies carried out by multiple facilities including JCMT, the $14 \mathrm{~m}$ telescope of the Purple Mountain Observation (PMO), and the $10.4 \mathrm{~m}$ telescope of the Caltech Submillimeter Observatory (CSO). The project was designed as a test of the JINGLE survey.

Galaxies were selected from the MaNGA MPL-3 (720 galaxies). The CO flux estimation for the MPL-3 galaxies also made use of the $L_{12 \mu \mathrm{m}}-M_{\mathrm{H}_{2}}$ relation as described in Section 2.1.1. A sample of 31 galaxies was selected for observations. The redshift range of these galaxies is 0.02-0.04. The galaxies were assigned to the various telescopes listed above according to the required sensitivity and the sensitivities of the telescopes. Some galaxies were observed by multiple telescopes to obtain both ${ }^{12} \mathrm{CO}(1-0)$ and ${ }^{12} \mathrm{CO}(2-1)$ data. The multiple transitions can be used to trace the physical conditions (e.g., temperature and density) of molecular gas.

There were 21 galaxies assigned to be observed by JCMT at ${ }^{12} \mathrm{CO}(2-1)$. The observations were done with the RxA receiver $^{28}$ between 2015 March and 2015 November, with a typical sky opacity of $\sim 0.12-0.32$. A total of 17 galaxies were assigned to the PMO $14 \mathrm{~m}$ telescope at ${ }^{12} \mathrm{CO}(1-0)$. Observations were carried out in the winter of 2015 with the nine-beam receiver. The beam size of PMO observations at $115 \mathrm{GHz}$ is $52^{\prime \prime}$, which can cover the entire galaxy at the typical redshift of the sample. Three galaxies were observed by the CSO at ${ }^{12} \mathrm{CO}(2-1)$ with the Heterodyne receiver in 2015 February. The beam size of the CSO at $230 \mathrm{GHz}$ is $30^{\prime \prime}$. The full design of the project and details of the data reduction are presented in Y. Gao et al. (2018, in preparation).

From a total of 31 galaxies in the JINGLE Pilot sample, we identify five additional galaxies in pairs for our analysis based on the criteria described in Section 2.1.1, with two from JCMT, two from PMO, and one from CSO observations. Of these, four are selected based on $r_{p}$ and $\Delta V$ and one based on Galaxy Zoo classification.

\subsubsection{Pair Sample: $x C O L D$ GASS}

We also include galaxies in pairs from the Extended CO Legacy Database for GASS (xCOLD GASS; Saintonge et al. 2017), an

\footnotetext{
${ }^{27}$ For reference, the number of galaxies in the first $\mathrm{CO}$ data release will be 72 (T. Xiao et al. 2018, in preparation).

${ }^{28}$ Observations taken at $230 \mathrm{GHz}$ at the JCMT prior to 2015 December were taken with RxA. Observations taken after this date (specifically, after 2016 May 13) are observed with a replacement mixer. The JCMT thus calls the new instrument RxA3m.
} 
extension of the IRAM $30 \mathrm{~m}$ legacy survey COLD GASS (Saintonge et al. 2011) that studies the molecular gas of nearby late-type galaxies with stellar masses $10<\log \left(M_{*} / M_{\odot}\right)<11.5$ and redshift $0.0025<z<0.05$. The xCOLD GASS survey extends the sample to $\log \left(M_{*} / M_{\odot}\right)=9.0$. COLD GASS and xCOLD GASS observe galaxies in ${ }^{12} \mathrm{CO}(1-0)$ and ${ }^{12} \mathrm{CO}(2-1)$ with IRAM and complementary observations from APEX ${ }^{12} \mathrm{CO}(2-1)$. The ${ }^{12} \mathrm{CO}(1-0)$ data are used for this work because the beam size is exactly the same as for the JCMT $15 \mathrm{~m}$ telescope at $230 \mathrm{GHz}$.

We identify 27 galaxies in pairs from all 532 xCOLD GASS galaxies. The criteria for selecting galaxies in pairs are the same as described in Section 2.1.1 $\left(r_{p}<50 \mathrm{kpch}^{-1}\right.$ and $\Delta V<$ $500 \mathrm{~km} \mathrm{~s}^{-1}$, or $P$-merger $>0.4$ and P-CS $>0.6$ ). A total of 27 galaxies in pairs are identified, and all of them are identified through the $r_{p}$ and $\Delta V$ criteria.

We have checked whether the galaxies from various observations reach different depths in terms of sensitivity. The main difference between the sample selection for the PI program/JINGLE/JINGLE Pilot and xCOLD GASS is the sensitivity (integration time) estimation. The former use the value of SFR $/ M_{\mathrm{H}_{2}}$, while the latter uses $M_{\mathrm{H}_{2}} / M_{*}$. The $M_{\mathrm{H}_{2}} / M_{*}$ limit is $2.5 \%$ for xCOLD GASS (Saintonge et al. 2017). For the galaxies from the PI programs, JINGLE, and JINGLE Pilot, the typical $M_{\mathrm{H}_{2}} / M_{*}$ achieved is $3.3 \%$, assuming $R_{21}=0.6$, $\alpha_{\mathrm{CO}}=4.3$ (as the values used in Section 2.1.1), and a common line width of $300 \mathrm{~km} \mathrm{~s}^{-1}$. We thus conclude that the samples analyzed here have comparable depth.

\subsubsection{Pair Sample: Summary}

In summary, our sample consists of a total of 58 galaxies in pairs (Table 2), of which 21 are from the JCMT PI programs, five from JINGLE, five from the JINGLE Pilot program, and 27 from xCOLD GASS. Fifty-six galaxies are selected based on $r_{p}$ and $\Delta V$ and two (one from the PI program and one from the JINGLE Pilot program) based on Galaxy Zoo morphologies. It is important to note that the Galaxy Zoo classification could potentially pick up galaxies in the post-coalescence stage (i.e., post-merger). This is not the case for the two galaxies identified through their morphologies; in other words, our sample does not contain post-mergers. We refer the reader to Ellison et al. (2013, 2018), Thorp et al. (2019), and M. Sargent et al. (2018, in preparation) for the star formation and cold gas properties of post-mergers.

For the two galaxies identified through Galaxy Zoo morphologies, we estimate their $r_{p}$ by calculating the distance between the two galactic nuclei. The distributions of galaxy properties are shown in Figure 1. Their merger properties are presented in Figure 2 (open and hatched histograms).

\subsection{Control Sample: $x C O L D$ GASS}

In order to quantify the effect of galaxy interactions on star formation and molecular gas properties, isolated galaxies are used as a comparison sample to our galaxies in pairs. Isolated galaxies are selected from xCOLD GASS and are systems that have no spectroscopic companion within $r_{p}<50 \mathrm{kpch}^{-1}$ and $\Delta V<500 \mathrm{~km} \mathrm{~s}^{-1}$ and a $P$-merger value equal to zero. We use the same criteria to control the morphology of the control sample as described in Section 2.1.1. Combining these criteria yields a control sample pool of 154 galaxies. About $8 \%$ of the selected control galaxies have no detection in ${ }^{12} \mathrm{CO}(1-0)$. An (a) (b)
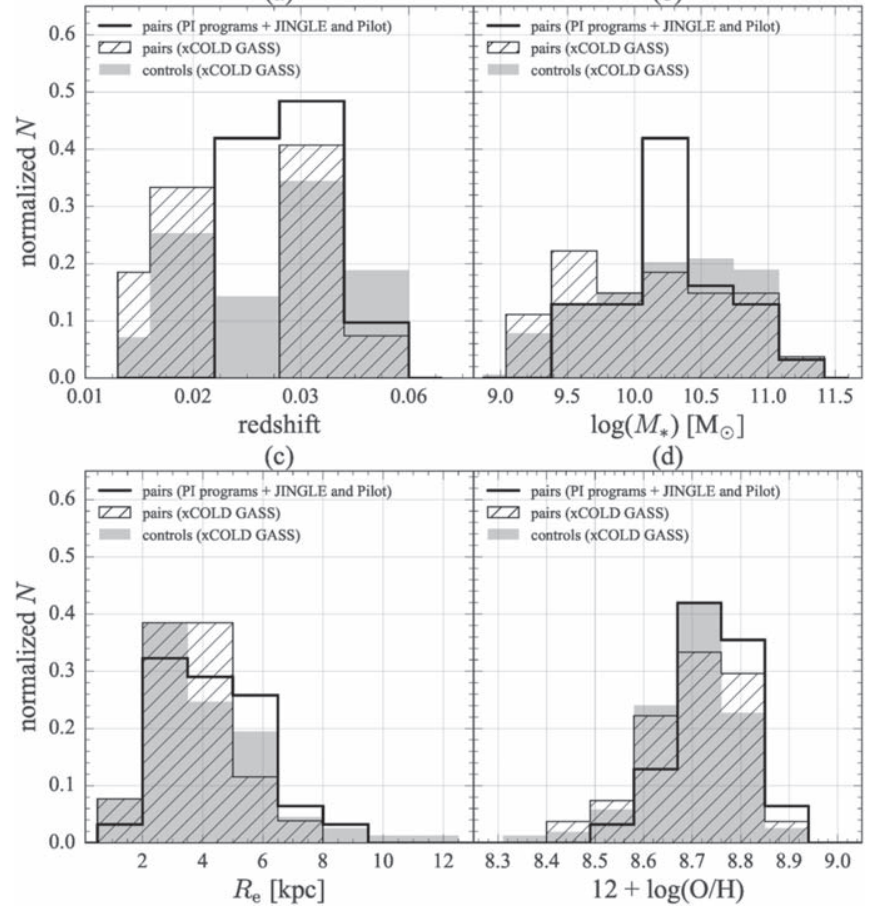

Figure 1. Histograms showing the distribution of the sample galaxies in terms of (a) redshift, (b) stellar mass, (c) effective radius measured at the $r$ band, and (d) gas-phase metallicity. The open histograms represent galaxies in pairs from the PI programs, JINGLE, and JINGLE Pilot. The hatched histograms show the galaxies in pairs from the xCOLD GASS survey. The pool of controls is shown as filled histograms.

upper limit for the flux of $3 \sigma$ is given for these galaxies (Saintonge et al. 2017). Since the integration limit $M_{\mathrm{H}_{2}} / M_{*}$ is as deep as $2.5 \%$ for xCOLD GASS, these nondetected galaxies thus truly have a lower gas fraction compared to other galaxies. These galaxies with upper limits for $L_{\mathrm{CO}}$ are included in the analysis. All of our conclusions remain unchanged if we use only galaxies with detections. The distributions of the galaxy properties of controls are shown in Figure 1 (filled histograms).

\subsection{Aperture Correction}

Some of the galaxies have optical sizes in excess of the telescope beams, so an aperture correction is required to correct the measured $\mathrm{CO}$ fluxes and turn them into estimates for the total flux. For reference, the range in the effective radius $\left(R_{\mathrm{e}}\right.$; Petrosian half-light radius measured at the $r$ band) of our sample is $\sim 2-6 \mathrm{kpc}$, as shown in Figure 1(c), corresponding to about $4^{\prime \prime}-12^{\prime \prime}$ at the redshifts of our sample (Figure 1(a)).

Aperture corrections are applied to all galaxies in pairs and control galaxies in this study. We adopt the method of Saintonge et al. (2012). For each galaxy, we create a model galaxy having an exponential molecular gas distribution with a profile following that of the stellar light. This assumption is based on the observation that $\mathrm{CO}$ and SFR distributions trace each other well in nearby galaxies (Leroy et al. 2009). Then, the model is convolved with a Gaussian matching the properties of the telescope beams. The aperture correction is the ratio between the total flux of the model and the flux in the beam area. The median aperture correction to the CO luminosity across the galaxies in pairs and the pool of controls are 0.09 and 0.08 dex, receptively. 
Table 2

Physical Properties of Galaxies in Pairs

\begin{tabular}{|c|c|c|c|c|c|c|c|c|c|c|}
\hline $\begin{array}{l}\text { Source } \\
\text { (1) }\end{array}$ & $\begin{array}{l}z \\
(2)\end{array}$ & $\begin{array}{c}\log \left(M_{*}\right) \\
\left(M_{\odot}\right) \\
(3)\end{array}$ & $\begin{array}{c}\log (\mathrm{SFR}) \\
\left(M_{\odot} \mathrm{yr}^{-1}\right) \\
(4)\end{array}$ & $\begin{array}{c}R_{\mathrm{e}} \\
(\mathrm{kpc}) \\
(5)\end{array}$ & $\begin{array}{c}r_{p} \\
(\mathrm{kpc}) \\
(6)\end{array}$ & $\begin{array}{l}\mu \\
(7)\end{array}$ & $\begin{array}{c}L_{\mathrm{CO}} / 10^{8} \\
\left(\mathrm{~K} \mathrm{~km} \mathrm{~s}^{-1} \mathrm{pc}^{2}\right) \\
(8)\end{array}$ & $\begin{array}{c}12+\log (\mathrm{O} / \mathrm{H}) \\
(9)\end{array}$ & $\begin{array}{c}\alpha_{\mathrm{CO}} \\
\left(M_{\odot} / L_{\mathrm{CO}}\right) \\
(10)\end{array}$ & $\begin{array}{c}\text { Parent } \\
\text { Sample } \\
(11)\end{array}$ \\
\hline $\mathrm{J} 130125.07+284038.0$ & 0.029 & 10.23 & 0.33 & 8.3 & 27.88 & 1.76 & $7.39(0.49)$ & 8.82 & 2.22 & $\mathrm{~J}$ \\
\hline $\mathrm{J} 130615.12+252737.9$ & 0.024 & 10.12 & 0.37 & 6.1 & 16.81 & 0.63 & 7.71(0.91) & 8.76 & 2.85 & $\mathrm{~J}$ \\
\hline $\mathrm{J} 132035.40+340821.7$ & 0.023 & 10.29 & 0.82 & 8.6 & 31.61 & 1.28 & $89.94(1.0)$ & 8.71 & 3.62 & $\mathrm{~J}$ \\
\hline $\mathrm{J} 132443.68+323225.0$ & 0.04 & 10.84 & 1.07 & 9.0 & 60.18 & 0.88 & $29.42(2.97)$ & 8.75 & 3.07 & $\mathrm{~J}$ \\
\hline $\mathrm{J} 133457.27+340238.7$ & 0.024 & 10.56 & 0.64 & 12.4 & 40.94 & 0.19 & $30.91(2.23)$ & 8.86 & 1.91 & $\mathrm{~J}$ \\
\hline J075641.84+175928.2 & 0.041 & 10.57 & 1.03 & 4.9 & 26.61 & 0.87 & $14.16(1.46)$ & 8.76 & 3.0 & $\mathrm{xCG}$ \\
\hline J081115.92+251045.7 & 0.014 & 9.62 & -0.38 & 7.8 & 29.08 & 0.74 & $0.52(0.08)$ & 8.65 & 4.25 & $\mathrm{xCG}$ \\
\hline J081905.10+214729.0 & 0.015 & 10.08 & 0.11 & 62.0 & 62.12 & 0.62 & $54.17(0.44)$ & 8.69 & 3.73 & $\mathrm{xCG}$ \\
\hline J084256.38+133829.7 & 0.017 & 9.68 & 0.31 & 12.4 & 4.95 & 0.04 & $2.3(0.21)$ & 8.7 & 3.83 & $\mathrm{xCG}$ \\
\hline J085254.99+030908.4 & 0.035 & 10.28 & -0.04 & 4.0 & 63.95 & 1.1 & $1.58(0.32)$ & 8.61 & 5.02 & $\mathrm{xCG}$ \\
\hline J090311.25+100907.0 & 0.03 & 10.11 & 0.3 & 9.6 & 51.6 & 0.53 & $5.83(0.58)$ & 8.84 & 2.11 & $\mathrm{xCG}$ \\
\hline J093236.58+095025.9 & 0.049 & 10.86 & 0.67 & 5.1 & 71.02 & 0.04 & $9.77(1.35)$ & 8.52 & 7.29 & $\mathrm{xCG}$ \\
\hline J095324.56+074956.2 & 0.039 & 10.67 & -0.36 & 4.2 & 61.17 & 0.82 & $2.44(0.54)$ & 8.68 & 3.51 & $\mathrm{xCG}$ \\
\hline $\mathrm{J} 103333.43+115216.9$ & 0.034 & 10.59 & 0.76 & 5.4 & 12.99 & 0.63 & $16.39(1.46)$ & 8.79 & 2.61 & $\mathrm{xCG}$ \\
\hline $\mathrm{J} 104024.66+065137.7$ & 0.03 & 10.89 & 0.07 & 6.8 & 68.8 & 0.31 & $8.91(0.78)$ & 8.55 & 6.11 & $\mathrm{xCG}$ \\
\hline $\mathrm{J} 112746.27+265734.5$ & 0.033 & 10.6 & -0.94 & 4.6 & 63.77 & 1.64 & $2.02(0.41)$ & 8.62 & 4.22 & $\mathrm{xCG}$ \\
\hline J112946.35+152001.1 & 0.037 & 11.02 & -0.76 & 6.3 & 70.29 & 1.64 & $4.88(0.74)$ & 8.67 & 3.32 & $\mathrm{xCG}$ \\
\hline J113116.03+043908.7 & 0.033 & 10.09 & 0.47 & 7.1 & 47.21 & 1.07 & $6.51(0.65)$ & 8.87 & 1.92 & $\mathrm{xCG}$ \\
\hline $\mathrm{J} 113701.89+153414.1$ & 0.013 & 9.88 & -0.52 & 15.4 & 45.25 & 1.44 & $1.84(0.16)$ & 8.76 & 2.71 & $\mathrm{xCG}$ \\
\hline J113914.72+145932.7 & 0.014 & 9.64 & -0.36 & 5.1 & 47.96 & 1.21 & $0.58(0.08)$ & 8.71 & 3.4 & $\mathrm{xCG}$ \\
\hline $\mathrm{J} 115020.17+255742.7$ & 0.013 & 9.36 & -0.21 & 11.7 & 30.97 & 0.88 & $0.49(0.07)$ & 8.44 & 10.33 & $\mathrm{xCG}$ \\
\hline J115726.68+251359.0 & 0.015 & 9.37 & -0.73 & 7.1 & 48.58 & -1.15 & $0.45(0.09)$ & 8.76 & 2.75 & $\mathrm{xCG}$ \\
\hline $\mathrm{J} 120222.51+295142.3$ & 0.01 & 9.98 & -0.16 & 8.8 & 55.07 & 0.76 & $5.91(0.5)$ & 8.77 & 2.69 & $\mathrm{xCG}$ \\
\hline J120409.73+014933.5 & 0.017 & 9.67 & -0.21 & 10.5 & 31.51 & -0.9 & $1.18(0.18)$ & 8.8 & 2.41 & $\mathrm{xCG}$ \\
\hline J125905.29+273839.9 & 0.018 & 9.67 & 0.09 & 6.6 & 40.87 & -0.26 & $1.98(0.21)$ & 8.82 & 2.32 & $\mathrm{xCG}$ \\
\hline $\mathrm{J} 130750.80+031140.7$ & 0.039 & 11.12 & -0.42 & 7.9 & 43.51 & 2.03 & $7.41(0.78)$ & 8.7 & 3.04 & $\mathrm{xCG}$ \\
\hline $\mathrm{J} 134701.23+335336.9$ & 0.017 & 9.78 & -0.31 & 16.0 & 26.29 & 0.29 & $1.21(0.15)$ & 8.64 & 4.46 & $\mathrm{xCG}$ \\
\hline $\mathrm{J} 135655.41+140832.1$ & 0.015 & 9.31 & -0.81 & 9.5 & 59.52 & 0.9 & $0.64(0.11)$ & 8.64 & 4.28 & $\mathrm{xCG}$ \\
\hline $\mathrm{J} 142342.38+340032.4$ & 0.013 & 9.84 & -0.02 & 8.4 & 14.95 & -0.18 & $2.46(0.2)$ & 8.77 & 2.72 & $\mathrm{xCG}$ \\
\hline J143525.34+002003.5 & 0.035 & 10.2 & 0.71 & 3.7 & 66.43 & 0.89 & $12.2(1.11)$ & 8.79 & 2.66 & $\mathrm{xCG}$ \\
\hline $\mathrm{J} 225258.55+010833.3$ & 0.016 & 9.5 & -0.7 & 10.3 & 58.37 & -0.87 & $0.58(0.08)$ & 8.6 & 5.12 & $\mathrm{xCG}$ \\
\hline $\mathrm{J} 231229.22+135632.1$ & 0.034 & 10.91 & -0.49 & 5.4 & 51.67 & 0.37 & $8.23(0.92)$ & 8.67 & 3.51 & $\mathrm{xCG}$ \\
\hline J025057.46+002209.8 & 0.044 & 10.05 & 0.63 & 3.1 & 26.08 & -0.13 & $3.34(0.79)$ & 8.8 & 2.58 & PI \\
\hline J031943.04+003355.7 & 0.024 & 10.06 & 0.27 & 7.6 & 44.48 & -0.75 & $1.83(0.66)$ & 8.73 & 3.19 & PI \\
\hline J032043.18-010008.2 & 0.036 & 10.64 & 0.68 & 5.5 & 12.15 & 1.14 & $35.82(1.69)$ & 8.75 & 2.97 & PI \\
\hline J032247.22+000857.7 & 0.023 & 10.38 & -1.29 & 13.7 & 32.19 & 1.18 & $6.77(0.77)$ & 8.67 & 3.38 & PI \\
\hline $\mathrm{J} 075454.46+535046.5$ & 0.035 & 10.76 & 0.7 & 6.0 & 60.07 & 1.69 & $21.41(1.21)$ & 8.72 & 3.4 & PI \\
\hline J082150.16+453110.6 & 0.054 & 10.38 & 0.77 & 3.4 & 59.25 & 0.73 & $14.95(1.02)$ & 8.85 & 2.07 & PI \\
\hline J093846.17+483346.3 & 0.025 & 9.43 & 0.11 & 5.4 & 20.88 & -0.94 & $3.1(0.43)$ & 8.71 & 3.6 & PI \\
\hline $\mathrm{J} 100508.31+443050.5$ & 0.026 & 10.36 & -0.74 & 9.6 & 65.66 & 0.38 & $3.65(1.37)$ & 8.66 & 3.75 & PI \\
\hline $\mathrm{J} 100718.98+463247.1$ & 0.024 & 10.17 & 0.03 & 7.1 & 37.5 & 1.44 & $5.14(0.72)$ & 8.82 & 2.17 & PI \\
\hline J102843.06+395019.9 & 0.029 & 9.95 & 0.45 & 3.5 & 38.56 & 0.53 & $4.22(1.25)$ & 8.82 & 2.37 & PI \\
\hline $\mathrm{J} 102855.10+395341.3$ & 0.044 & 10.26 & -0.0 & 6.2 & 27.37 & 0.5 & $13.15(1.07)$ & 8.71 & 3.32 & PI \\
\hline $\mathrm{J} 121049.28+443045.3$ & 0.023 & 10.18 & -0.12 & 5.3 & 41.8 & 1.5 & $1.16(0.14)$ & 8.73 & 3.09 & PI \\
\hline $\mathrm{J} 130420.70+450323.9$ & 0.028 & 9.66 & 0.34 & 7.2 & 62.3 & 0.63 & $5.07(0.67)$ & 8.65 & 4.6 & PI \\
\hline $\mathrm{J} 134109.43+231640.5$ & 0.027 & 9.88 & -0.16 & 3.1 & 13.98 & -0.66 & $12.21(1.31)$ & 8.75 & 2.94 & PI \\
\hline $\mathrm{J} 135129.47+434823.1$ & 0.033 & 10.16 & 0.61 & 4.4 & 43.84 & 1.33 & $6.77(1.0)$ & 8.71 & 3.62 & PI \\
\hline $\mathrm{J} 140057.82+425120.3$ & 0.032 & 10.67 & 0.49 & 6.0 & 25.33 & 0.0 & 13.17(1.04) & 8.76 & 2.84 & PI \\
\hline $\mathrm{J} 153545.82+445005.2$ & 0.03 & 10.19 & 0.47 & 8.8 & 49.31 & 1.03 & $8.9(1.35)$ & 8.78 & 2.66 & PI \\
\hline $\mathrm{J} 154219.34+475636.7$ & 0.037 & 9.65 & -0.36 & 3.7 & 15.87 & -0.33 & $12.28(0.79)$ & 8.67 & 3.94 & PI \\
\hline $\mathrm{J} 160242.58+411150.1$ & 0.033 & 10.41 & 0.69 & 7.8 & 17.46 & 0.42 & $22.59(2.2)$ & 8.82 & 2.31 & PI \\
\hline J163349.62+391547.5 & 0.032 & 10.74 & 0.42 & 6.2 & 32.83 & 1.01 & $10.68(0.8)$ & 8.73 & 3.1 & PI \\
\hline J172823.84+573243.4 & 0.029 & 9.61 & 0.8 & 3.9 & 7.79 & $\ldots$ & $5.48(0.81)$ & 8.51 & 8.47 & PI \\
\hline J032042.95-010631.1 & 0.021 & 10.02 & -0.04 & 11.6 & 55.22 & 0.9 & $3.92(0.26)$ & 8.79 & 2.47 & $\mathrm{JP}$ \\
\hline J074637.71+444725.8 & 0.031 & 11.16 & 0.2 & 9.3 & 33.58 & 0.6 & $39.69(2.53)$ & 8.67 & 3.8 & JP \\
\hline J091500.75+420127.8 & 0.028 & 10.27 & 0.87 & 4.9 & 61.02 & -0.35 & $9.48(1.85)$ & 8.76 & 3.01 & JP \\
\hline J091555.53+441957.9 & 0.04 & 10.95 & 1.23 & 10.4 & 9.73 & $\ldots$ & $46.21(4.58)$ & 8.78 & 2.81 & JP \\
\hline J110637.36+460219.5 & 0.025 & 10.44 & 0.52 & 4.3 & 27.03 & 1.76 & 14.81(1.65) & 8.77 & 2.76 & JP \\
\hline
\end{tabular}

Note. Column (1) SDSS ID; column (2) SDSS spectroscopic redshift; column (3) stellar mass from the MPA-JHU Catalog (Section 2.4); column (4) SFR from the MPA-JHU Catalog (Section 2.4); column (5) NSA 50\% light radius measured at the $r$ band; column (6) projected separation between two galaxies in a pair; column (7) stellar mass ratio between two galaxies in a pair (Section 2.5); column (8) aperture- and line ratio-corrected $\left(R_{21}=0.8\right) \mathrm{CO}$ luminosity $L_{\mathrm{CO}}$ and its uncertainty (Section 2.3); column (9) gas-phase metallicity (Section 2.6); column (10) CO-to- $\mathrm{H}_{2}$ conversion factor (Section 3.1); column (11) parent samples (Section 2.1), where $\mathrm{J}=\mathrm{JINGLE}$ (Section 2.1.2), $\mathrm{xCG}=\mathrm{xCOLD}$ GASS (Section 2.1.4), PI = PI programs (Section 2.1.1), and JP = JINGLE Pilot (Section 2.1.3). 
(a)

(b)

(c)

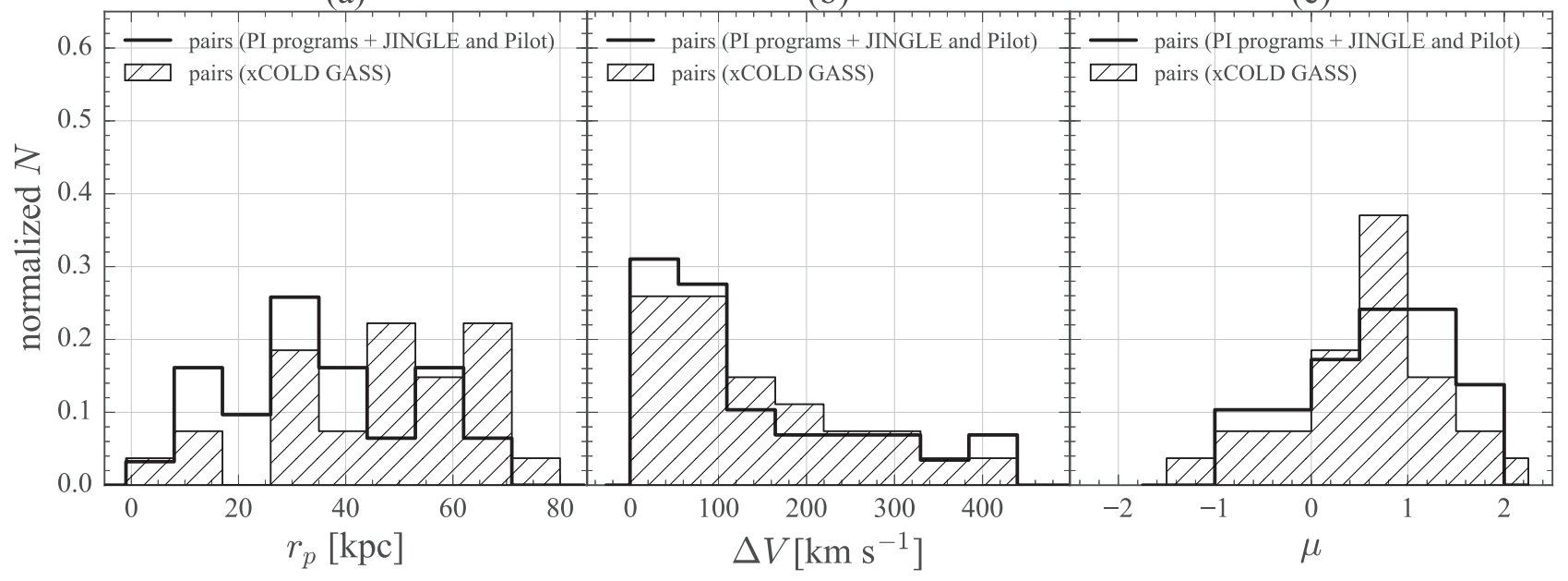

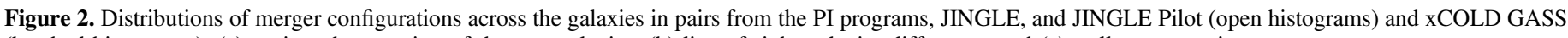
(hatched histograms): (a) projected separation of the two galaxies, (b) line-of-sight velocity difference, and (c) stellar mass ratio.

\subsection{Global Stellar Mass and SFR}

The global SFR and $M_{*}$ are taken from the MPA-JHU DR7 public catalog. ${ }^{29}$ The MPA-JHU catalog assumes a Kroupa IMF (Kroupa 2001). Here $M_{*}$ is estimated by fitting stellar population models from Bruzual \& Charlot (2003) to the ugriz SDSS photometry, following the method of Kauffmann et al. (2003a). The $M_{*}$ values have been found to agree with other estimates (e.g., Taylor et al. 2011; Mendel et al. 2014; Chang et al. 2015). To estimate SFRs, Brinchmann et al. (2004) first distinguished the emission-line properties based on the theoretical upper (lower) limit for pure starburst (AGN) models (Kewley et al. 2001; Kauffmann et al. 2003b) on the BaldwinPhillips-Terlevich (BPT) diagram (Baldwin et al. 1981). For galaxies in which the primary source of ionizing photons is from H II regions, SFRs are estimated by fitting a grid of photoionization models from Charlot \& Longhetti (2001) to the observed $\mathrm{H} \alpha, \mathrm{H} \beta,[\mathrm{O}$ III] , and [N II] line fluxes. These SFR estimates agree well with those derived from the infrared fluxes (Charlot et al. 2002). For galaxies falling outside of the starforming regime on the BPT diagrams, since the line fluxes might be affected by the AGN component, their SFRs are estimated based on the relation between specific SFR $\left(\mathrm{sSFR}=\mathrm{SFR} / M_{*}\right)$ and $\mathrm{D} 4000$ (Brinchmann et al. 2004). This relationship was constructed using the sSFR and D4000 of starforming galaxies. According to the BPT diagram, the pair sample consists of eight AGNs, 11 composites, and 39 starforming galaxies. The pool of the control sample includes 31 AGNs, 34 composites, and 89 star-forming galaxies. It is worth noting that by adopting currently popular modified-BPT diagrams to distinguish Seyfert and low-ionization emissionline (nuclear) regions (LI(N)ERs) in the AGN regime (Cid Fernandes et al. 2013), the majority of the AGNs in our sample (six out of eight for galaxies in pairs and 27 of the controls) are LI(N)ERs, which could in fact be powered by stellar populations instead of a nuclear compact source (Belfiore et al. 2016; Hsieh et al. 2017).

The aperture corrections are determined for these central SFR measurements by fitting the photometry of the outer regions of the galaxies (Salim et al. 2007). In the following analysis, the median SFR and $M_{*}$ from the probability

\footnotetext{
${ }^{29}$ https://wwwmpa.mpa-garching.mpg.de/SDSS/DR7/\#derived
}

distributions for each galaxy are used. Using the average SFR and $M_{*}$ does not change the results.

The distribution of the pair sample in the SFR versus $M_{*}$ plane is shown in Figure 3 with colored symbols. Galaxies taken from the control sample drawn from xCOLD GASS are represented by gray squares. Gray dots are the remaining xCOLD GASS targets not selected as control, for reference.

\subsection{Mass Ratio of Pairs}

Pairs in this work contain both primary (higher- $M_{*}$ galaxy in a pair) and secondary (lower- $M_{*}$ galaxy) galaxies. For each pair, we define the mass ratio as the stellar mass of the COobserved galaxy divided by that of its companion and take the logarithm of the ratio $(\mu)$. A positive value of $\mu$ implies that the observed galaxy is the primary galaxy in the pair, and vice versa. A few companions do not have the MPA-JHU measurement for $M_{*}$. In this case, the mass ratios of these systems are calculated using $M_{*}$ from the NASA-Sloan Atlas catalog ${ }^{30}$ for both galaxies. It is not possible to derive the mass ratio for the two pairs identified through Galaxy Zoo morphologies, as the two galaxies in the pairs are too close to have separated measurements. These two galaxies appear to be major mergers, and the $\mathrm{CO}$ observations are made toward the primary galaxies. They are excluded from the discussion involving mass ratio. The distribution of the mass ratio is shown in Figure 2(c).

\subsection{Gas-phase Metallicity $12+\log (\mathrm{O} / \mathrm{H})$}

In this work, we adopt a metallicity-dependent $\alpha_{\mathrm{CO}}$ (see Section 3.1). Gas-phase metallicity is calculated using the O3N2 method empirically calibrated by Pettini \& Pagel (2004):

$$
12+\log (\mathrm{O} / \mathrm{H})=8.73-0.32\left(\frac{[\mathrm{O} \mathrm{III]}}{\mathrm{H} \beta} / \frac{[\mathrm{N} \mathrm{III}]}{\mathrm{H} \alpha}\right) .
$$

The emission-line fluxes are obtained from the MPA-JHU DR7 release. ${ }^{31}$ All of the emission-line fluxes of our galaxies in pairs and controls have $\mathrm{S} / \mathrm{Ns}$ higher than 3.5 if the flux uncertainties in

\footnotetext{
30 http://nsatlas.org/

31 https://wwwmpa.mpa-garching.mpg.de/SDSS/DR7/raw_data.html
} 


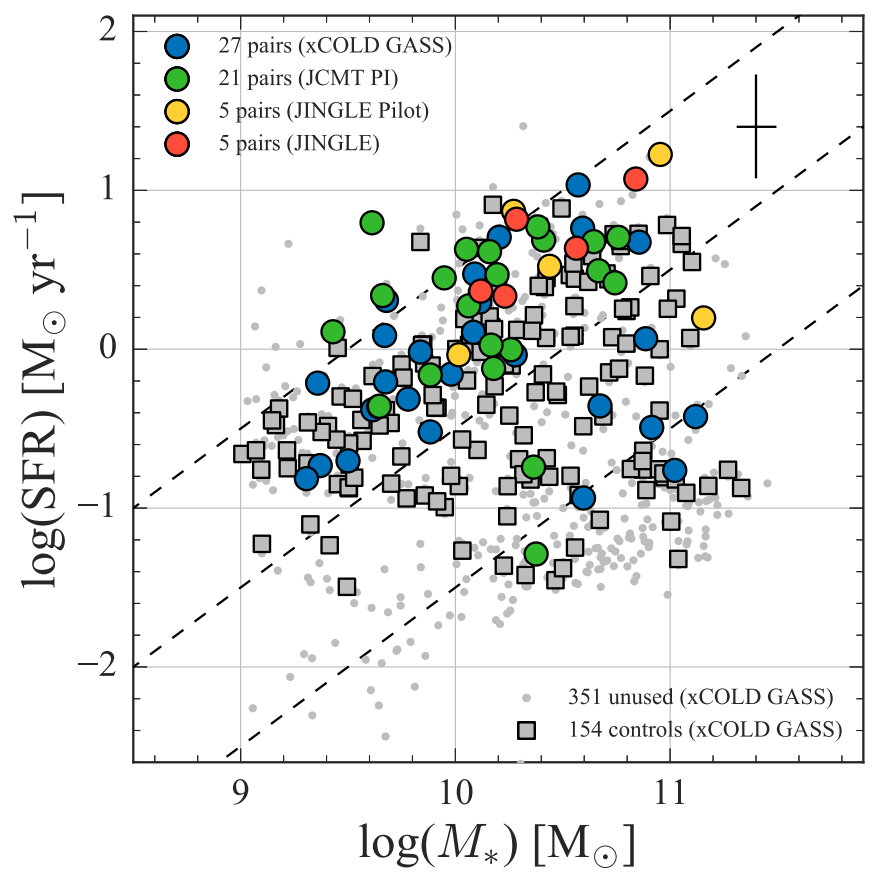

Figure 3. Global SFR vs. stellar mass relation of galaxies discussed in this work. Galaxies in pairs used in this work are shown as colored symbols. Galaxies from the control sample are shown as gray squares. Those in the xCOLD GASS sample that are not used in the analysis are shown as gray dots (see text for details). The SFR and $M^{*}$ values are taken from the MPA-JHU catalog. From top to bottom, the three dashed lines correspond to $\log \left(\mathrm{sSFR} / \mathrm{yr}^{-1}\right)=-9.5,-10.5$, and -11.5 .

the catalog are used or higher than 2 (mostly $\gg 5$ ) if the scaling factors $^{32}$ provided in the MPA-JHU DR7 website are applied to the flux uncertainties, which were calculated by the comparisons of the derived line fluxes of galaxies that were observed multiple times. Since these emission lines are close in wavelength, dust extinction should have a minimal effect on the fluxes. Galaxies in pairs and controls have similar ranges of metallicity, from 8.3 to 8.9 . The median values are 8.7 for both populations (Figure 1(d)).

\section{Results}

\subsection{SFR and Gas Properties in the Full Pair Sample}

To get an idea of the distribution of galaxy properties within our sample, we first show the normalized distribution of galaxy $\mathrm{sSFR}, L_{\mathrm{CO}}$, and gas properties. In cases where the $\mathrm{CO}$ line is undetected, the $3 \sigma$ upper limits of $L_{\mathrm{CO}}$ luminosity (Section 2.2) are used for the analysis and plots in this paper.

Figure 4(a) shows the distribution of sSFR in the pair sample as a filled histogram and for the control sample as an open histogram. While the two distributions peak at the same sSFR, the median sSFR of galaxies in pairs $\left(\log \left(\mathrm{sSFR} / \mathrm{yr}^{-1}\right)=-9.9\right)$ is higher than that of isolated galaxies $\left(\log \left(\mathrm{sSFR} / \mathrm{yr}^{-1}\right)=\right.$ $-10.3)$. We check whether the distributions in Figure 4(a) are sampled from the same parent distribution. This is tested with the Kolmogorov-Smirnov (K-S) statistic, whose results are listed in Table 3. The K-S test returns a $p$-value $=1.9348 \times$ $10^{-5}$, suggesting a $>99.9 \%$ probability that the sSFRs of

\footnotetext{
32 The scaling factors are 2.473, 1.882, 1.566, and 2.039 for the $\mathrm{H} \alpha, \mathrm{H} \beta$, [O III], and [N II] lines, respectively.
}

galaxies in pairs and controls are two distinct distributions. This is in agreement with the many previous studies that have found enhanced star formation in galaxies in pairs (e.g., Di Matteo et al. 2007; Ellison et al. 2008; Scudder et al. 2012; Patton et al. 2013; Knapen et al. 2015). In Sections 3.3 and 3.4, we return to SFR differences as a function of projected separation and mass ratio, respectively.

Turning now to gas properties, in Figure 5(a), we first show the comparison of the integrated $L_{\mathrm{CO}}$ of galaxies in pairs and controls. From this figure, it is evident that galaxies in pairs (filled histogram) show higher $L_{\mathrm{CO}}$ compared to the pool of controls (open histogram). The difference is significant. The K$\mathrm{S}$ test results in a $p$-value of 0.0017 .

After having looked at the observed quantity $L_{\mathrm{CO}}$, we now derive the physical quantities of gas from the measurements. The distribution of $M_{\mathrm{H}_{2}}$ is shown in Figure 4(b). Here $L_{\mathrm{CO}}$ is converted to $M_{\mathrm{H}_{2}}$ by multiplying by the conversion factor, $\alpha_{\mathrm{CO}}$. The value for $\alpha_{\mathrm{CO}}$ is calculated following Accurso et al. (2017), in which $\alpha_{\mathrm{CO}}$ is metallicity-dependent, with a secondorder dependence on the offset of a galaxy from the starforming main sequence,

$$
\begin{aligned}
\log \alpha_{\mathrm{CO}}( \pm 0.165)= & 15.623-1.732[12+(\mathrm{O} / \mathrm{H})] \\
& +0.051 \log \Delta(\mathrm{MS}),
\end{aligned}
$$

where $\Delta(\mathrm{MS})$ is the distance off the analytical definition of the main sequence by Whitaker et al. (2012). No significant difference in the ranges and median values of $\alpha_{\mathrm{CO}}$ is found between galaxies in pairs and controls. The derived $\alpha_{\mathrm{CO}}$ values are in the range of $1.9-10.3 M_{\odot}\left(\mathrm{K} \mathrm{km} \mathrm{s}^{-1} \mathrm{pc}^{2}\right)$ for the galaxies in pairs (Table 2) and 2.1-16.5 $M_{\odot}\left(\mathrm{K} \mathrm{km} \mathrm{s}^{-1} \mathrm{pc}^{2}\right)$ for the controls. The median $\alpha_{\mathrm{CO}}$ values are 3.0 and $3.2 M_{\odot}$ $\left(\mathrm{K} \mathrm{km} \mathrm{s}^{-1} \mathrm{pc}^{2}\right)$ for galaxies in pairs and controls, respectively. The distributions of $M_{\mathrm{H}_{2}}$ for the galaxies in pairs and controls largely overlap, but the peak $M_{\mathrm{H}_{2}}$ of galaxies in pairs is higher than that of controls. The median $\log \left(M_{\mathrm{H}_{2}} / M_{\odot}\right)$ values of galaxies in pairs and controls are 9.3 and 8.9, respectively. According to the K-S test, the differences between galaxies in pairs and controls are real: the probability that their $M_{\mathrm{H}_{2}}$ values are from the same distribution is less than $1 \%$ ( $p$-value $=$ 0.0016).

The $f_{\text {gas }}$ and SFE are calculated based on the derived $M_{\mathrm{H}_{2}}$. The gas fraction $f_{\text {gas }}$ is defined as

$$
f_{\text {gas }}=\frac{M_{\mathrm{H}_{2}}}{M_{\mathrm{H}_{2}}+M_{*}} .
$$

Note that some studies adopt the definition of $f_{\text {gas }}=M_{\mathrm{H}_{2}} / M_{*}$. The two definitions are approximately the same when $M_{\mathrm{H}_{2}} \ll M_{*}$ (i.e., low $f_{\text {gas }}$ ). Our results would be qualitatively the same if we adopted this definition. Figure 4(c) shows the normalized distribution of the molecular gas fraction. The $\log \left(f_{\text {gas }}\right)$ of the controls (open histogram) spans the range -2.37 to 0.64 (corresponding to $0.4 \%-22.9 \%$ ) with a median value of $-1.26(5.5 \%)$. The values are consistent with other studies of galaxies in the local universe (e.g., Leroy et al. 2008; Bothwell et al. 2009; Saintonge et al. 2017). On the other hand, the range of $\log \left(f_{\text {gas }}\right)$ of galaxies in pairs (filled histogram) shifts to larger values, from -1.81 to $-0.20(1.5 \%-63.1 \%)$, with a median value of $-1.03(9.3 \%)$. A K-S test of $f_{\text {gas }}$ yields a 

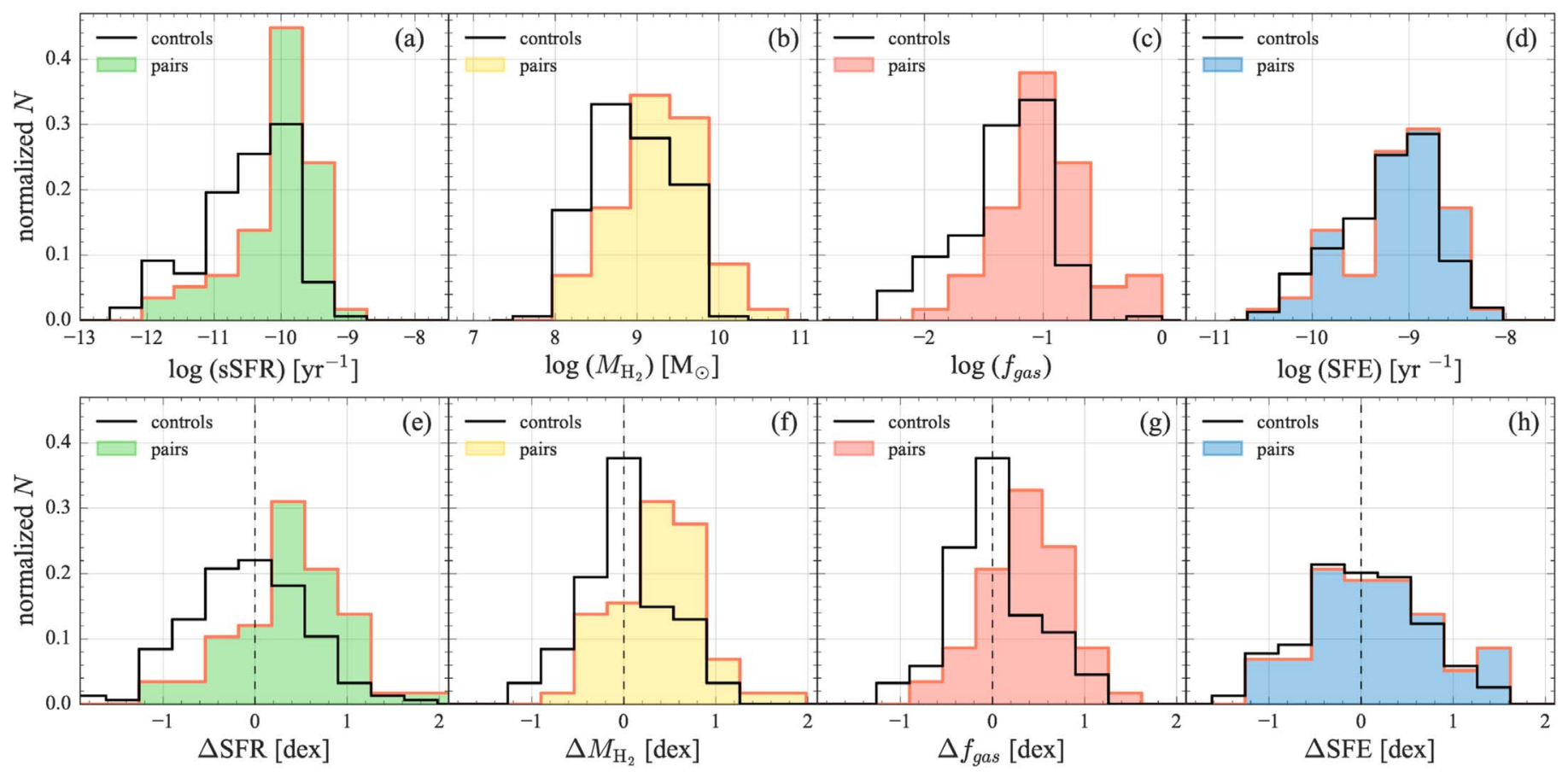

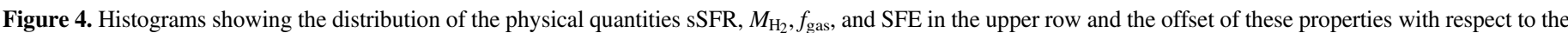

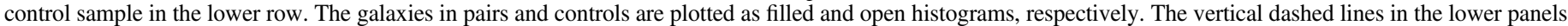

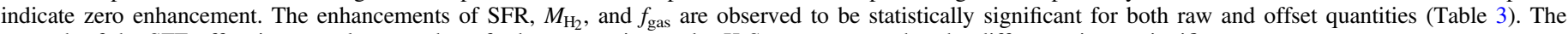
strength of the SFE offset is not as large as that of other properties, and a K-S test suggests that the difference is not significant.

Table 3

Summary of the K-S Test Results for Galaxies in Pairs and Controls

\begin{tabular}{lcc}
\hline \hline & $\begin{array}{c}\text { Raw Value } \\
(\text { Section 3.1) }\end{array}$ & $\begin{array}{c}\text { Offset Value }(\Delta) \\
(\text { Section 3.2) }\end{array}$ \\
\hline$(\mathrm{s}) \mathrm{SFR}$ & $1.9348 \times 10^{-5}$ & $8.2130 \times 10^{-6}$ \\
$L_{\mathrm{CO}}$ & 0.0017 & $4.3003 \times 10^{-5}$ \\
$M_{\mathrm{H}_{2}}$ & 0.0016 & $1.1890 \times 10^{-6}$ \\
$f_{\text {gas }}$ & $3.9535 \times 10^{-7}$ & $8.7772 \times 10^{-7}$ \\
SFE & 0.2128 & 0.4806 \\
\hline
\end{tabular}

$p$-value $=3.9535 \times 10^{-7}$. There is less than a $0.1 \%$ chance that the two samples come from the same distribution.

The SFE is defined as

$$
\frac{\mathrm{SFE}}{\left(\mathrm{yr}^{-1}\right)}=\frac{\mathrm{SFR}}{M_{\mathrm{H}_{2}}}
$$

and is shown in Figure 4(d). The ranges and peaks of SFE appear closely matched for galaxies in pairs and controls. Median $\log \left(\mathrm{SFE} / \mathrm{yr}^{-1}\right)$ values are -9.02 and -9.13 for galaxies in pairs and controls, respectively. The values of SFE are comparable with those of other studies of nearby isolated galaxies (e.g., Leroy et al. 2008; Koyama et al. 2017). A K-S test suggests that the two samples are drawn from the same distribution $(p$-value $=0.2128)$.

We have shown in this section that there are statistically significant differences in SFR, $M_{\mathrm{H}_{2}}$, and $f_{\text {gas }}$ between the galaxies in pairs and the full control sample, and that there is no significant difference in SFE between these two populations. However, we have not yet considered possible differences in the fundamental properties of these two samples, such as stellar mass and redshift. Since the SFR and gas properties themselves depend on these underlying characteristics, a rigorous

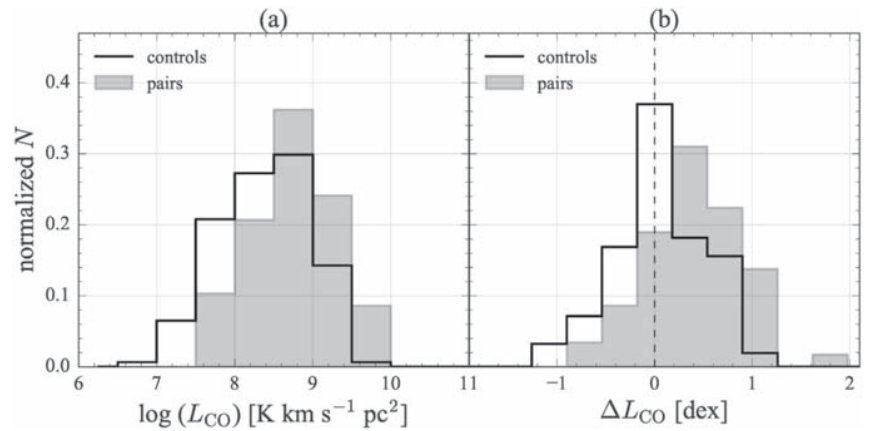

Figure 5. Distributions of (a) $L_{\mathrm{CO}}$ and (b) $\Delta L_{\mathrm{CO}}$. The galaxies in pairs and controls are plotted as filled and open histograms, respectively. The vertical dashed line in panel (b) indicates zero enhancement. The figures confirm that the enhancement of the $M_{\mathrm{H}_{2}}$ and $f_{\text {gas }}$ of gas in galaxies in pairs is already indicated from the comparison from the observed quantity $L_{\mathrm{CO}}$, i.e., before the conversion to $M_{\mathrm{H}_{2}}$.

comparison requires a careful matching between the galaxies in pairs and the control sample. We investigate this in the following section.

\subsection{Offset of SFR and Gas Properties}

In order to fairly compare the properties of the galaxies in pairs and controls on a galaxy-by-galaxy basis, and accounting for dependences on properties such as redshift and $M_{*}$, we compute "offset" quantities in this section. Our approach closely follows that of Violino et al. (2018) for $\mathrm{H}_{2}$ fractions in a smaller sample of galaxies in pairs, as well as that of Ellison et al. (2018) for H I fractions in post-mergers. Each galaxy, including pairs and controls, is matched in redshift, stellar mass, and effective radius with a minimum of five control galaxies from the pool of controls. The tolerance of stellar 
(a)

(b)

(c)

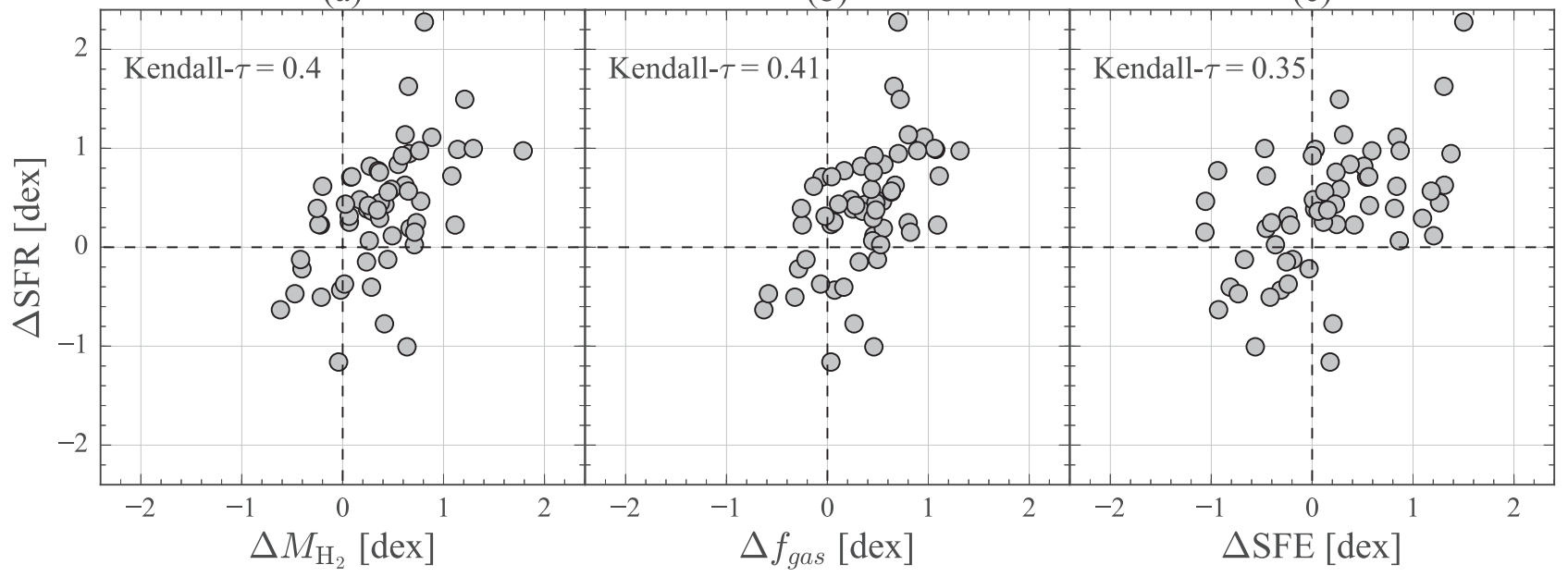

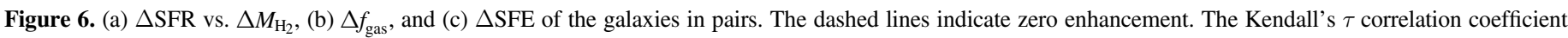

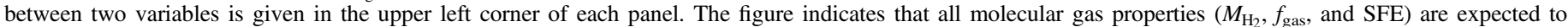
influence SFR.

mass, redshift, and effective radius are $0.1 \mathrm{dex}, 0.005$, and $25 \%$, respectively. The criteria are allowed to grow by 0.1 dex, 0.005 , and $5 \%$, respectively, until the minimum required number of control galaxies is reached. Most of the galaxies could find sufficient control galaxies in the first round. The "offset" of a galaxy property $(P)$ is defined as

$$
\Delta(P)=\log \left(P_{\text {gal }}\right)-\log \left(\text { median }\left(P_{\text {control }}\right)\right),
$$

where $P_{\text {gal }}$ is the property of the galaxy in question and median $\left(P_{\text {control }}\right)$ is the median property of its control galaxies. We should emphasize that, as this is taken in the logarithm form, it really is a ratio of the value of the galaxy in question against the median value of its controls. A positive offset represents an enhancement, while a negative value implies a suppression of the property. Distributions of offset properties are presented in Figure 5(b) for $L_{\mathrm{CO}}$ and Figures 4(e)-(h) for other physical quantities related to $\mathrm{SFR}^{33}$ and gas properties. Open and filled histograms denote the offset properties of controls and galaxies in pairs, respectively. The median values for the controls are around zero, as expected, the width showing the intrinsic spread of the quantities plotted.

The enhancement of SFR is still present and significant $\left(p\right.$-value $=8.2130 \times 10^{-6}$; see Table 3$)$, as indicated by the peaks of the distributions (Figure 4(e)). The median $\triangle$ SFR is 0.40 dex (a factor of $\sim 2.5$ enhancement), confirming the wellknown fact that, statistically, galaxy-galaxy interaction enhances the SFR, but not dramatically so; moreover, there is a large spread in the enhancement of SFR (e.g., Scudder et al. 2012; Knapen et al. 2015).

Figure 5(b) confirms that the enhancement of the amount of gas in galaxies in pairs is already indicated from the comparison from the observed $L_{\mathrm{CO}}$, i.e., before the conversion to $M_{\mathrm{H}_{2}}$. The K-S test yields a $p$-value of $4.3003 \times 10^{-5}$. The median $\Delta L_{\mathrm{CO}}$ of galaxies in pairs is 0.40 dex.

\footnotetext{
33 In Section 3.1, we use "sSFR" to express the absolute value of SFR so that the dependence of SFR on $M_{*}$ is considered. The offset property of star formation is defined as the difference of "SFR" between galaxies in pairs and controls. The two SFRs are not different, as the $M_{*}$ is matched when selecting the control sample to calculate the SFR offset.
}

The median $\Delta M_{\mathrm{H}_{2}}$ and $\Delta f_{\text {gas }}$ are enhanced by similar factors, about 0.37 and 0.44 dex, respectively (Figures $4(\mathrm{f})$ and (g)). We also apply the K-S test to the offset distributions of galaxies in pairs and controls. For $M_{\mathrm{H}_{2}}$ and $f_{\text {gas }}$, the K-S test gives a $<1 \%$ probability of the two distributions being drawn from the same parent distribution (Table 3).

The strength of the SFE offset is not as large as that of other properties. The median $\triangle$ SFE implies an offset by 0.14 dex with respect to the controls. However, a K-S test suggests that the difference is not significant; there is a high probability that the two distributions (control and pairs) are drawn from the same population.

In order to investigate which gas property is most strongly correlated with SFR enhancement, in Figure 6 we plot $\Delta f_{\text {gas }}$, $\Delta M_{\mathrm{H}_{2}}$ and $\Delta \mathrm{SFE}$ versus $\Delta \mathrm{SFR}$. All gas property offsets increase with $\Delta$ SFR. We use Kendall's $\tau$ correlation coefficient to quantify the strength of the dependence. The computation yields positive correlations of $0.40,0.39$ and 0.35 for $\Delta M_{\mathrm{H}_{2}}$, $\Delta f_{\text {gas }}$ and $\Delta \mathrm{SFE}$, respectively. Based on this figure, we can speculate that all molecular gas properties $\left(M_{\mathrm{H}_{2}}, f_{\mathrm{gas}}\right.$, and $\left.\mathrm{SFE}\right)$ are expected to influence SFR.

Figure 7 presents the $\Delta$ SFE versus $\Delta f_{\text {gas }}$, color-coded by $\Delta$ SFR. The large and small circles represent galaxies in pairs and controls, respectively. Various inferences can be drawn from this figure. The highest $\Delta$ SFRs predominantly occur in galaxies with both enhanced $\Delta f_{\text {gas }}$ and enhanced $\Delta \mathrm{SFE}$; however, enhanced $\Delta f_{\text {gas }}$ and $\Delta$ SFE together do not always result in a high magnitude of $\Delta$ SFR (but almost all galaxies with positive values of $\Delta f_{\text {gas }}$ and $\Delta$ SFE show enhanced SFR). Galaxies may not have enhanced SFR if only $f_{\text {gas }}$ or SFE is enhanced. Finally, galaxies associated with both suppressed SFE and $f_{\text {gas }}$ are likely to have suppressed SFR as well.

\section{3. $\triangle S F R, \triangle \mathrm{f}_{\text {gas }}$, and $\triangle S F E$ as a Function of Projected Separation}

Figure $8(\mathrm{a})$ presents the $\triangle \mathrm{SFR}$ of our galaxies in pairs as a function of $r_{p}$. Gray circles denote individual galaxies in pairs, and colored squares show the mean $\Delta \mathrm{SFR}$ at different $r_{p}$ bins. Error bars are obtained by calculating the sample standard deviation and dividing by $\sqrt{N}$, where $N$ is the number of 


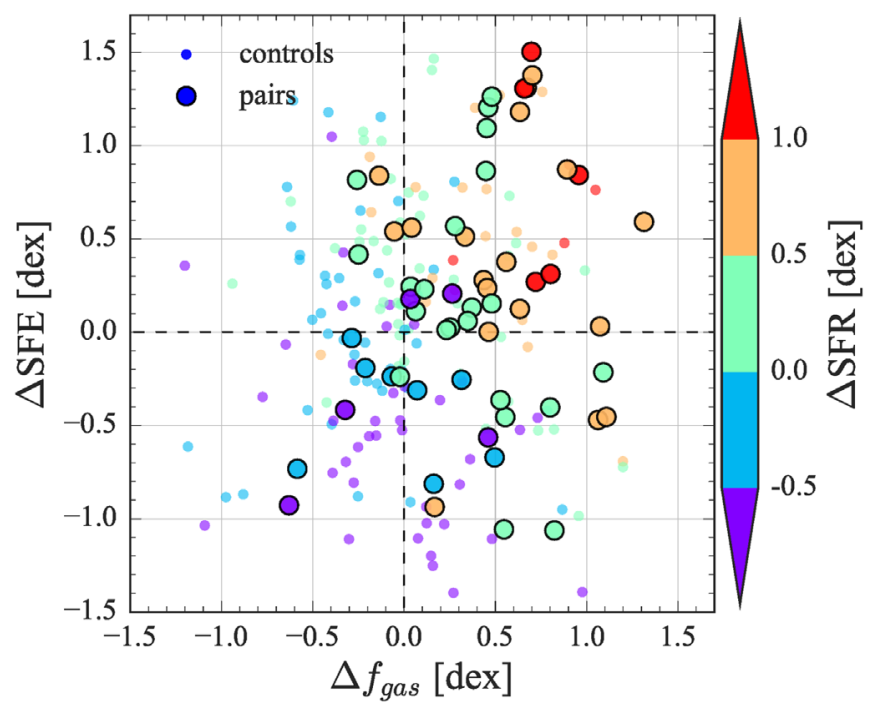

Figure 7. The $\Delta \mathrm{SFE}$ vs. $\Delta f_{\text {gas }}$, color-coded by $\Delta \mathrm{SFR}$. The large and small circles represent galaxies in pairs and controls, respectively. The highest $\Delta$ SFRs ( $>0.5$ dex) predominantly occur in galaxies with both enhanced $\Delta f_{\text {gas }}$ and enhanced $\Delta \mathrm{SFE}$; however, enhanced $\Delta f_{\text {gas }}$ and $\Delta \mathrm{SFE}$ together do not always result in a high magnitude of $\Delta$ SFR (but it is true that almost all galaxies with positive values of $\Delta f_{\text {gas }}$ and $\Delta$ SFE show enhanced SFR). Galaxies may not have enhanced SFR if only $f_{\text {gas }}$ or SFE is enhanced (upper left and lower right corners of the figure). Finally, galaxies associated with both suppressed SFE and $f_{\text {gas }}$ are likely to have suppressed SFR as well (lower left corner of the figure).

galaxies at each $r_{p}$ bin. The dashed horizontal line denotes zero enhancement. At small separations, galaxies in pairs are found to have substantially higher SFR than their controls, with median $\Delta$ SFR increasing from $\approx 0.2 \mathrm{dex}$ at $\sim 70 \mathrm{kpc}$ to $\sim 0.9$ dex at $<10 \mathrm{kpc}$. The fastest rise of $\Delta$ SFR occurs at $r_{p} \approx$ 20-30 kpc, as also shown in Scudder et al. (2012) and Patton et al. (2013) with much larger sample sizes than this work and in Bustamante et al. (2018) with cosmological simulations. The $\Delta$ SFR $(0.9 \pm 0.5$ dex $)$ in the lowest $r_{p}$ bin is higher than that of post-mergers $(0.5 \pm 0.1 \mathrm{dex})$ in Ellison et al. (2013). This may be due to a mix of post-mergers that are already quenched and actively forming stars, as the SFR and duration of enhanced SFR at the coalescence phase depend on various merger configurations (e.g., Di Matteo et al. 2007, 2008; Bustamante et al. 2018; Thorp et al. 2019).

The enhanced SFR as two galaxies approach each other could be interpreted as direct evidence of tidally triggered star formation. Park \& Choi (2009) investigated the dependence of galaxy properties on both the small- and large-scale environments. They found that galaxy properties, such as $\mathrm{H} \alpha$ equivalent width, surface brightness profile, and colors, abruptly change when $r_{p}$ corresponds to the $0.05 \times$ virial radius of the nearest-neighbor galaxy (see their Figures 6 and 7). This corresponds to $\sim 20 \mathrm{kpc}$ for the galaxies in their sample. This interpretation can be applied to our result of the boost of $\Delta \mathrm{SFR}$ at $r_{p} \approx 20-30 \mathrm{kpc}$. However, we should note that this characteristic radius depends on the stellar mass of the sample galaxies because the hydrodynamic interactions between galaxies depend on the stellar mass (Park \& Choi 2009).

It should be noted that there is significant scatter within each $r_{p}$ bin (see also Scudder et al. 2012). There are several reasons for the scatter. First, the peak in SFR enhancement does not always occur near coalescence. The SFR could reach the peak

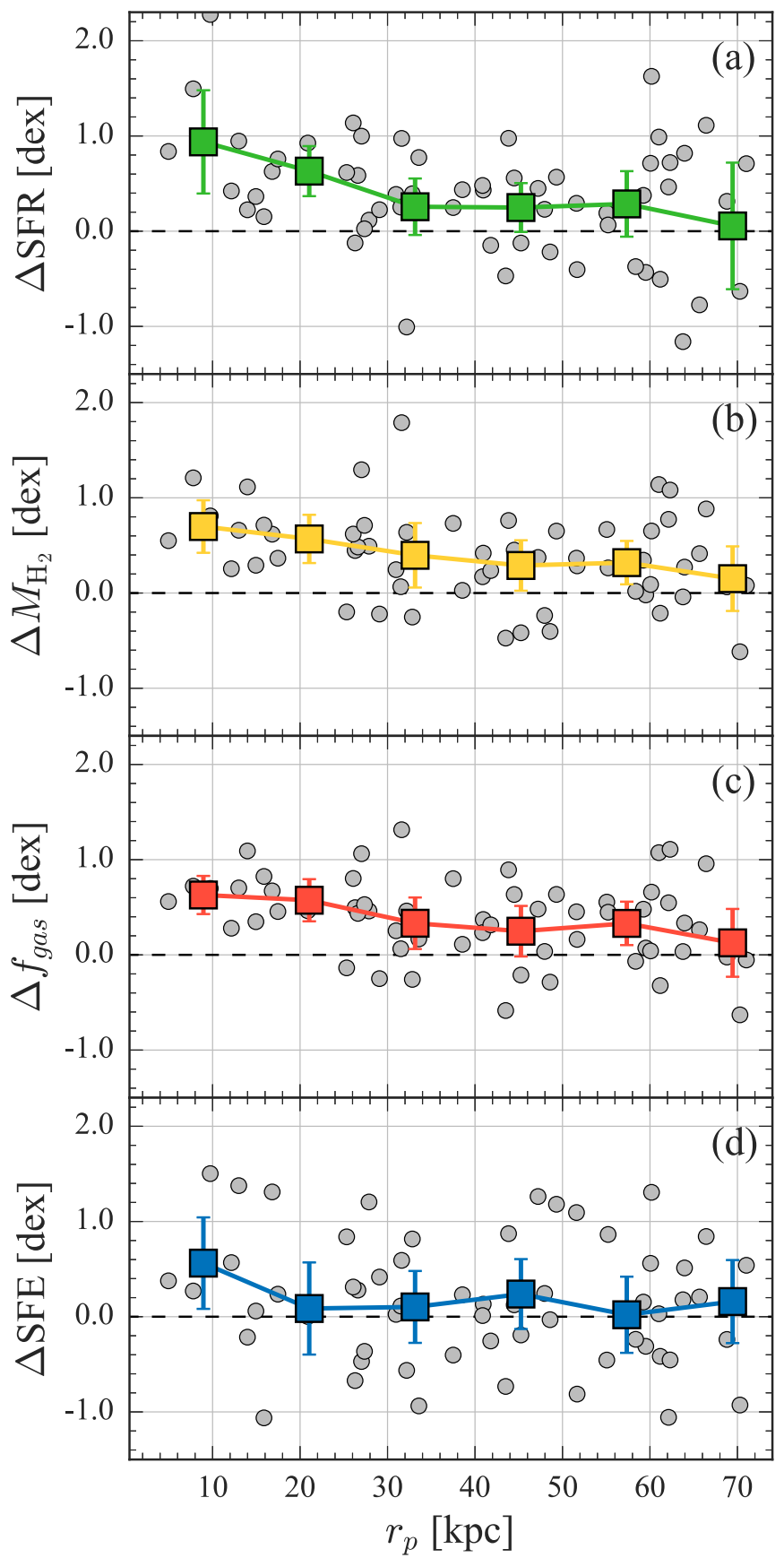

Figure 8. Offset properties as a function of projected galaxy separation for our sample. Gray circles denote individual galaxies. Mean values per $r_{p}$ are indicated with colored squares. Error bars are obtained by calculating the sample standard deviation and dividing by $\sqrt{N}$, where $N$ is the number of galaxies at each $r_{p}$ bin. The horizontal lines indicate no enhancement. The $\Delta \mathrm{SFR}, \Delta M_{\mathrm{H}_{2}}$, and $\Delta f_{\text {gas }}$ all increase with decreasing pair separation over the range from $\sim 70$ to $10 \mathrm{kpc}$. However, any SFE enhancement is only significant at the smallest pair separations.

when two galaxies are still several tens of kpc apart (Di Matteo et al. 2008; Sparre \& Springel 2016). Moreover, $\Delta$ SFR depends on the mass ratio. This will be discussed in Section 3.4. Many studies have stressed the importance of the properties of the companion in determining the SFR enhancement (Park \& Choi 2009; Hwang et al. 2010; Xu et al. 2012; Cao et al. 2016). Specifically, the SFRs of spirals in spiral-spiral pairs are more likely to be enhanced compared to the spirals in mixed spiral-elliptical pairs. The suppression (or 
zero enhancement) of star formation in the disks in the mixed pairs may be caused by the extended X-ray halos (i.e., hot gas) of an early-type companion of a spiral galaxy and prevent the spiral from forming stars, or there is no inflow of cold gas from the early-type companion (Park \& Choi 2009; Hwang et al. 2010). Furthermore, SFR enhancement seems to be correlated with the properties of the orbit of the two interacting galaxies, as shown by the simulations of Sparre \& Springel (2016), in which high-density gas preferentially appears in head-on mergers with very high collision velocities. This scenario is difficult to test directly by observation due to the ambiguity, even when detailed models can be constructed, in a system's geometry and orbital parameters. Finally, SFR enhancement is also found to correlate with $\mathrm{H}$ I fraction (e.g., Scudder et al. 2015). Since the galaxies in pairs used in this work are not restricted to any specific merger property, the scatter in each $r_{p}$ bin is somewhat expected. We should note that part of this scatter is also due to the fact that the $r_{p}$ is not a direct measure of the merging sequence because galaxies in pairs would merge after several encounters, and their orbital geometry is complicated. This presumably also introduces some extra scatter in the relation.

Turning to the gas properties, panels (b) and (c) present the change of $\Delta M_{\mathrm{H}_{2}}$ and $\Delta f_{\text {gas }}$ with $r_{p}$, respectively. All symbols are as defined for panel (a). Here $\Delta M_{\mathrm{H}_{2}}$ and $\Delta f_{\text {gas }}$ versus $r_{p}$ show very similar behavior to $\Delta \mathrm{SFR}$, and $\Delta M_{\mathrm{H}_{2}}$ and $\Delta f_{\text {gas }}$ gradually increase from $\sim 0$ dex at $70 \mathrm{kpc}$ to $\sim 0.7$ and $0.6 \mathrm{dex}$, respectively, at $<10 \mathrm{kpc}$. In particular, the figures show that almost all close pairs $\left(r_{p} \leqslant 25 \mathrm{kpc}\right)$ appear to have $M_{\mathrm{H}_{2}}$ and $f_{\text {gas }}$ enhancements.

Here we compare our results of $\Delta f_{\text {gas }}$ with other studies in which the offset of gas properties is also calculated. The 11 galaxies in pairs in Violino et al. (2018) have an $r_{p}$ range of $16-30 \mathrm{kpc}$. The median offset $M_{\mathrm{H}_{2}}$ and $f_{\text {gas }}$ of their galaxies in pairs are 0.34 and 0.40 dex, respectively. For our galaxies in pairs in the same $r_{p}$ range, the median $\Delta M_{\mathrm{H}_{2}}$ and $\Delta f_{\text {gas }}$ are 0.49 and 0.46 , respectively, slightly higher than that of Violino et al. (2018). It may simply be due to low number statistics. The degree of the $f_{\text {gas }}$ enhancement of our galaxies in pairs at short $r_{p}(\sim 0.6 \mathrm{dex})$ is consistent with that of the sample of postmergers (M. Sargent et al. 2018, in preparation).

The dependence of $\triangle \mathrm{SFE}$ on $r_{p}$ is different from that of $\Delta \mathrm{SFR}, \Delta M_{\mathrm{H}_{2}}$, and $\Delta f_{\text {gas }}$. Statistically, SFE enhancements only occur at the smallest pair separations $\left(r_{p}<20 \mathrm{kpc}\right)$ by $\sim 0.5$ dex. In other words, although overall, the $\triangle \mathrm{SFE}$ shows zero enhancement (Figure 4(h)), there is a systematic offset for the smallest $r_{p}$ galaxies. The scatter of $\triangle \mathrm{SFE}$ is large at large $r_{p}$. We will discuss the possible contribution of the large scatter later in the discussion section (Section 4.2).

The statistical significances of the correlations are assessed by calculating the Kendall's $\tau$ correlation coefficients. The correlation coefficients are $-0.33,-0.29$, and -0.29 for $r_{p}$ versus $\Delta \mathrm{SFR}, \Delta M_{\mathrm{H}_{2}}$, and $\Delta f_{\text {gas }}$, respectively. If we restrict the analysis to galaxies with $r_{p}<30 \mathrm{kpc}$, where the offset values appear to rise more rapidly with decreasing $r_{p}$, the correlation coefficients become $-0.40,-0.30$, and -0.31 for $\Delta S F R$, $\Delta M_{\mathrm{H}_{2}}$, and $\Delta f_{\text {gas }}$, respectively. The correlation coefficients suggest that there are only marginal anticorrelations between $\Delta \mathrm{SFR}, \Delta M_{\mathrm{H}_{2}}$, and $\Delta f_{\text {gas }}$ and the pair separation, possibly due to the large scatter at a fixed $r_{p}$. The absence of a correlation between $r_{p}$ and $\triangle \mathrm{SFE}$ is also suggested by the correlation coefficient of -0.10 across all galaxies in pairs. The

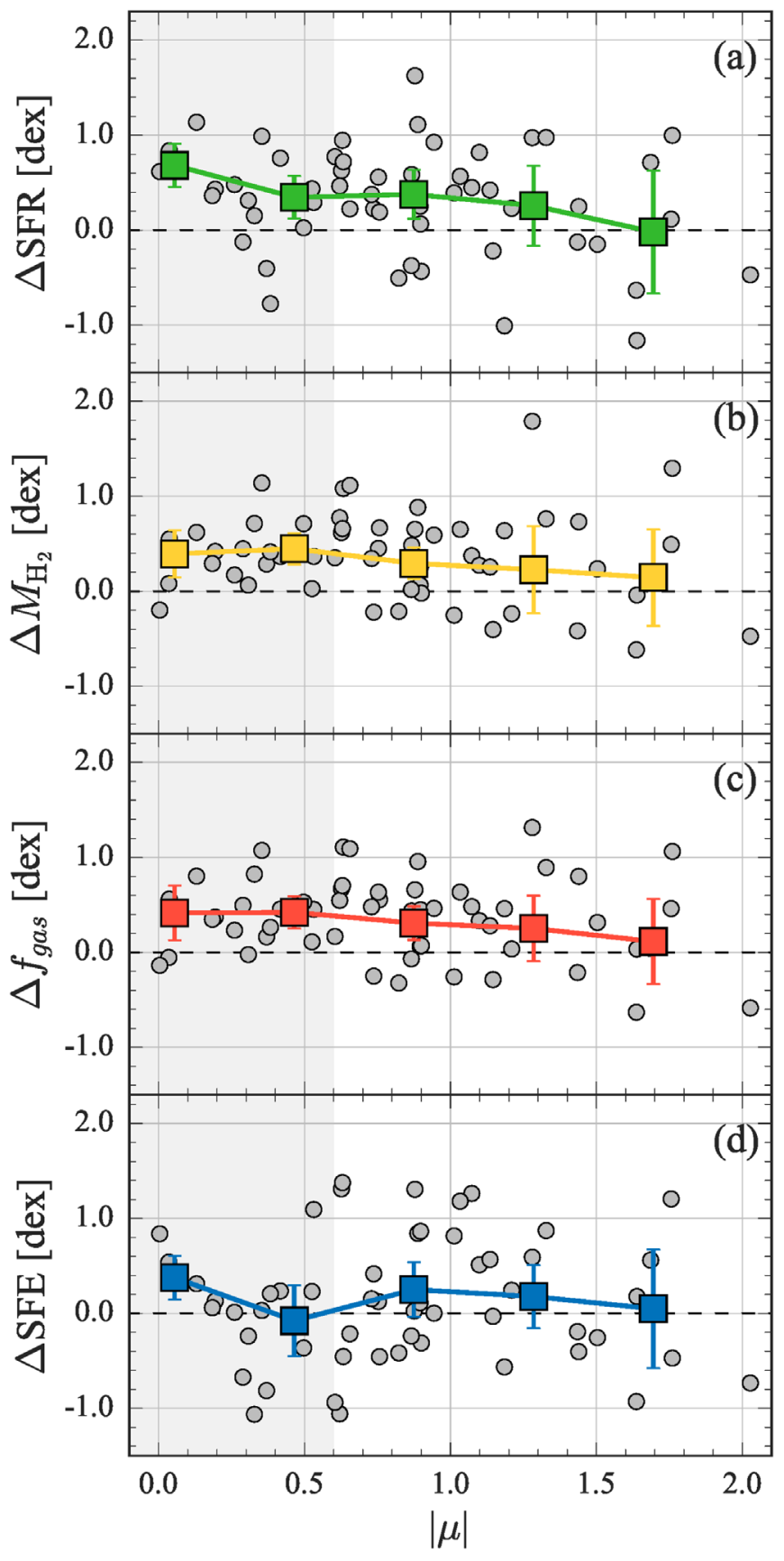

Figure 9. Offset properties as a function of the absolute value of stellar mass ratio $|\mu|$ of the galaxies in pairs. The major-merger regime $(|\mu|<0.6)$ is highlighted in gray. The individual galaxies in pairs are shown with gray circles, and the means are in colored symbols. The $\Delta \mathrm{SFR}, \Delta M_{\mathrm{H}_{2}}$, and $\Delta f_{\text {gas }}$ exhibit a trend with mass ratio of the two galaxies in a pair. We find no apparent dependence between the mass ratio and $\triangle \mathrm{SFE}$. Any SFE enhancement is only significant in the equal-mass pairs $(|\mu| \approx 0)$.

correlation coefficient of $r_{p}$ and $\triangle \mathrm{SFE}$ becomes -0.22 for galaxies with $r_{p}<30 \mathrm{kpc}$.

\section{4. $\triangle S F R, \triangle \mathrm{f}_{\text {gas }}$, and $\triangle S F E$ as a Function of Mass Ratio}

Our sample covers about a two orders of magnitude mass ratio and includes both primary and secondary galaxies. Since the number of secondary galaxies is considerably smaller than the primary galaxies (11 versus 45$)$, thus not allowing us to compare between these two populations, in this section, we 
consider the absolute value of mass ratio $|\mu|$. Figure 9(a) presents $\Delta$ SFR as a function of $|\mu|$. The major-merger regime $(|\mu|<0.6)$ is shown in gray. The individual galaxies in pairs are shown with gray circles, and the means are in colored symbols.

The mean $\Delta$ SFRs are progressively higher for smaller $|\mu|$ values. Most, but not all, major mergers in our sample show SFR enhancement. As suggested by simulations, a major merger is not inevitably accompanied by significant SFR enhancement, depending on the geometry of the collisions (Cox et al. 2008; Moreno et al. 2015; Sparre \& Springel 2016). This may explain why some of the major mergers show low $\triangle \mathrm{SFR}$.

The $\Delta M_{\mathrm{H}_{2}}$ and $\Delta f_{\text {gas }}$ exhibit a similar trend as $\Delta \mathrm{SFR}$ in Figures 9(b) and (c), increasing from large to low-mass ratio. On the other hand, the $\triangle \mathrm{SFE}$ trend with mass ratio is not as strong as for other properties. There is no significant difference in $\triangle$ SFE across $|\mu|$. Statistically, SFE enhancements only occur in the equal-mass pairs $(|\mu| \approx 0)$ by $\sim 0.4$ dex.

We also quantify the degree of correlations between the offset properties and $|\mu|$ using the Kendall's $\tau$ correlation coefficient. The correlation coefficients are $-0.25,-0.18$, -0.20 , and -0.001 for $\Delta \mathrm{SFR}, \Delta M_{\mathrm{H}_{2}}, \Delta f_{\text {gas }}$, and $\Delta \mathrm{SFE}$, respectively, indicating marginal trends for equal-mass pairs to have higher $\Delta \mathrm{SFR}, \Delta M_{\mathrm{H}_{2}}$, and $\Delta f_{\text {gas }}$ but not $\Delta \mathrm{SFE}$.

\section{Discussion}

\subsection{Are the Enhanced $\Delta M_{\mathrm{H}_{2}}$ and $\Delta f_{\text {gas }}$ Real?}

While several studies have suggested the enhancement of $\Delta M_{\mathrm{H}_{2}}$ and $\Delta f_{\text {gas }}$ in galaxies in pairs (e.g., Combes et al. 1994; Casasola et al. 2004; Violino et al. 2018), it remains unclear from where this mass excess originates. We first check whether the enhanced $\Delta M_{\mathrm{H}_{2}}$ and $\Delta f_{\text {gas }}$ are real.

The enhanced $\Delta M_{\mathrm{H}_{2}}$ and $\Delta f_{\text {gas }}$ could be the contamination from the $\mathrm{CO}$ emission of the companions. We check the possible contamination by comparing the projected separation between two galaxies in a pair and the beam size (radius). For galaxies with $r_{p}>30 \mathrm{kpc}$, the distance to the companion is well beyond the beam area, with a median distance of $8 \times$ the beam radius. For galaxies with $11<r_{p}<30 \mathrm{kpc}$, companions are located at $1.5-10 \times$ the beam radius away from the pointing of the $\mathrm{CO}$ observations; the median distance is $3 \times$ the beam radius. For the close pairs with $<11 \mathrm{kpc}$, the beam areas cover a part of the disk of their companions. In one of the cases, the nucleus of the companion falls within the beam area. Consequently, there must be a nonnegligible contribution of $\mathrm{CO}$ emission from the companions in these measurements. However, it is unlikely to be the sole cause of the enhanced $\Delta M_{\mathrm{H}_{2}}$ and $\Delta f_{\text {gas }}$, as the offsets are considerably larger than a factor of 2 (or $0.3 \mathrm{dex}$ ), and the positive offsets are seen in galaxies beyond the separation for contamination. Moreover, $\Delta f_{\text {gas }}$ enhancement is seen in post-mergers by $\sim 0.6 \mathrm{dex}$ (M. Sargent et al. 2018, in preparation), where there is only one galaxy in the beam. Therefore, an additional mechanism must be involved in boosting the amount of molecular gas. Nonetheless, we conservatively quote an overestimation (underestimation) of $0.3 \mathrm{dex}$, corresponding to $\sim 2$ times, for the derived $\Delta M_{\mathrm{H}_{2}}$ and $\Delta f_{\text {gas }}(\Delta \mathrm{SFE})$, assuming a situation consisting of a major merger where both galaxies fall within the beam area.
The observed enhanced $\Delta M_{\mathrm{H}_{2}}$ and $\Delta f_{\text {gas }}$ could partially be a selection effect toward high-SFR objects (and thus likely molecular gas-rich), since some of the pair sample (PI program, JINGLE, and JINGLE Pilot) are selected based reaching a minimum $\mathrm{S} / \mathrm{N}$ level of 4 in an on-source time shorter than 250 minutes that is estimated on the basis of observed SFR and the empirical SFR $-M_{\mathrm{H}_{2}}$ relation (Section 2). We cannot fully rule out the possibility that with deeper data, we would detect more galaxies in pairs with $\Delta M_{\mathrm{H}_{2}}$ and $\Delta f_{\text {gas }}$ comparable to that of the control sample. For example, a far-infrared $(\propto \mathrm{SFR})$ selection of galaxies in the Coma cluster prevented Casoli et al. (1991) from finding molecular gas-deficient galaxies in clusters, while $\mathrm{H}_{2}$-deficient galaxies are now widely found when other selection criteria are used, such as stellar mass (e.g., Boselli et al. 2014).

In order to consider such an effect, we additionally match the galaxies in pairs and controls in SFR with an initial tolerance of 0.1 dex and a step of 0.05 dex. In carrying out this test with the extra matching parameter, we find that it is difficult to have at least five control galaxies for each pair, due to the limited number of pool galaxies; thus, the minimal number of control galaxies is instead set to three. Moreover, we were unable to identify control galaxies for some galaxies in pairs with high sSFR; these objects are excluded from the analysis here. The results are presented in Figures 10(a)-(d).

With SFR as one of the matching parameters (Figure 10(a)), the enhancements of $\Delta M_{\mathrm{H}_{2}}$ and $\Delta f_{\text {gas }}$ are still present in galaxies in pairs, although with smaller values. The distributions of $\Delta M_{\mathrm{H}_{2}}$ and $\Delta f_{\text {gas }}$ when additionally matched in SFR are presented in Figures $10(\mathrm{~b})$ and (c). The median $\Delta M_{\mathrm{H}_{2}}$ and $\Delta f_{\text {gas }}$ become 0.19 and $0.11 \mathrm{dex}(\sim 55 \%$ and $30 \%)$, respectively. This reduction of the difference in molecular gas content between galaxies in pairs and controls when they additionally matched in SFR is also found by Violino et al. (2018). However, we note that the median values here should be treated as lower limits, since the galaxies in pairs with high sSFR are not considered, and these galaxies potentially have large $\Delta M_{\mathrm{H}_{2}}$ and $\Delta f_{\text {gas }}$.

After matching in SFR, SFE seems to be suppressed in galaxies in pairs. The median $\triangle \mathrm{SFE}$ is $-0.20 \mathrm{dex}$ (Figure $10(\mathrm{~d})$ ). This is not unexpected, since SFE is SFR (matched with controls) divided by $M_{\mathrm{H}_{2}}$ (enhanced) by definition.

In addition, we also perform another test to examine the potential bias induced by different selection criteria for galaxies in pairs (see Section 2), that is, only including galaxies from $x$ COLD GASS (without matching in SFR). In this case, the selection of a control sample uses exactly the same criteria as for galaxies in pairs. This would also remove any uncertainty in the conversion between $\mathrm{CO}(2-1)$ and $\mathrm{CO}(1-0)$ intensities. The results are shown in Figures 10(e)-(h) (specifically, these plots are made with the same data values that generated Figures 4(e) -(h), but only galaxies in pairs from xCOLD GASS are shown). It is evident that the distributions of $\Delta \mathrm{SFR}, \Delta M_{\mathrm{H}_{2}}$, and $\Delta f_{\text {gas }}$ still peak at higher values with respect to the controls when only the xCOLD GASS galaxies in pairs are considered. The median offset values are $\sim 0.3$ dex for $\Delta \mathrm{SFR}, \Delta M_{\mathrm{H}_{2}}$, and $\Delta f_{\text {gas }}$ and $\sim 0.1$ dex for $\Delta \mathrm{SFE}$.

Overall, we argue that the contamination from companions and selection effects are not the main causes of the enhanced $\Delta M_{\mathrm{H}_{2}}$ and $\Delta f_{\text {gas }}$. 

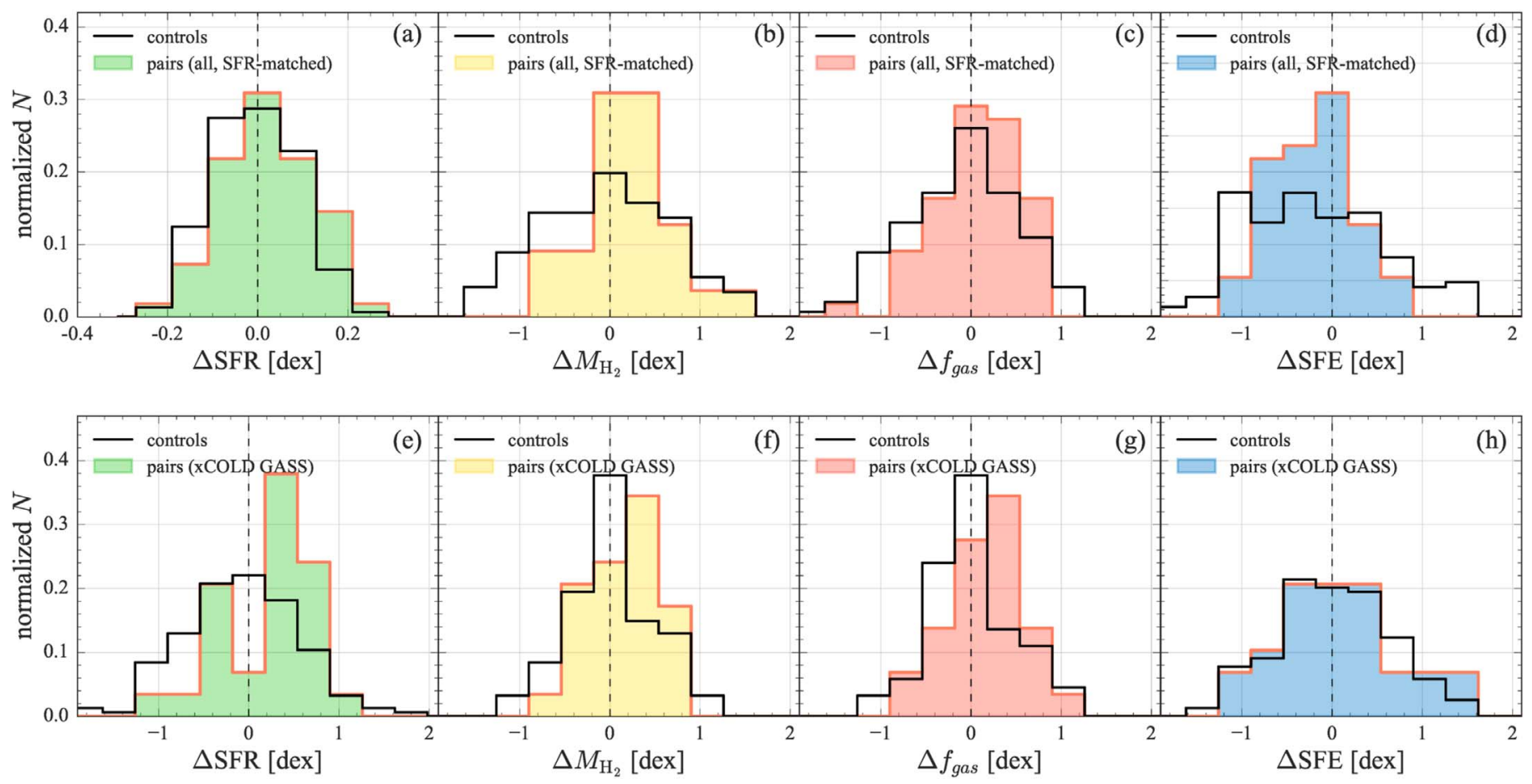

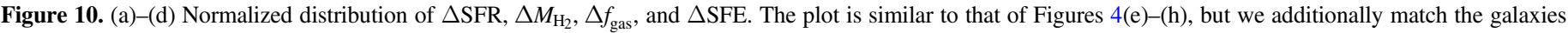

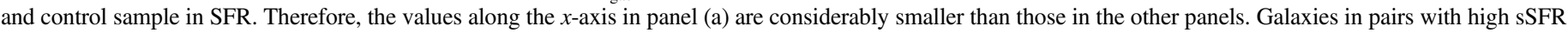

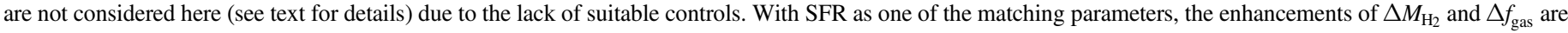

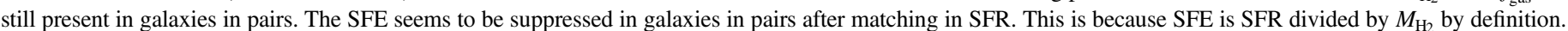

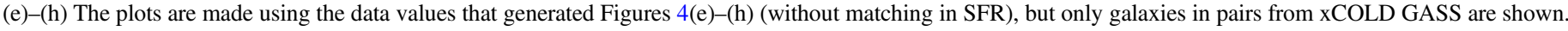

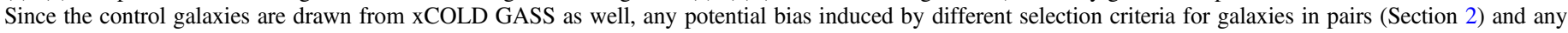

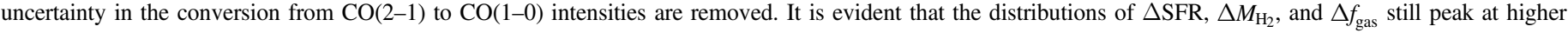
values with respect to the controls when only the xCOLD GASS galaxies in pairs are considered.

\subsection{What Drives the Gas Reservoir Enhancement?}

The main results of this work are that (1) there is a clear enhancement in the gas reservoir $\left(M_{\mathrm{H}_{2}}\right.$ and $\left.f_{\mathrm{gas}}\right)$ in galaxies in pairs (Section 3.2); (2) $M_{\mathrm{H}_{2}}$ and $f_{\text {gas }}$ increase by a similar degree to SFR, while the SFE is compatible with not being enhanced (Section 3.2); (3) the correlation with $\triangle \mathrm{SFR}$ is stronger for $\Delta M_{\mathrm{H}_{2}}$ and $\Delta f_{\text {gas }}$ than $\Delta \mathrm{SFE}$ (Section 3.2); and (4) the dependences of $\Delta M_{\mathrm{H}_{2}}$ and $\Delta f_{\text {gas }}$ on merger configurations are similar to that of $\Delta \mathrm{SFR}$, with $\Delta \mathrm{SFR}, \Delta M_{\mathrm{H}_{2}}$, and $\Delta f_{\text {gas }}$ increasing with decreasing $r_{p}$ and $|\mu|$, whereas $\Delta \mathrm{SFE}$ is not exhibiting any trend, with only close pairs $\left(r_{p}<10 \mathrm{kpc}\right)$ and equal-mass systems $(|\mu| \approx 0)$ being affected significantly (Section 3.3).

The results of our work are in broad agreement with several previous studies. Combes et al. (1994) also found a correlation of SFR and molecular gas mass (both are in raw values) with $r_{p}$. At the same time, they found that the SFE shows no evidence for a correlation with $r_{p}$ and is only enhanced in galaxies displaying the strongest distortion, presumably analogous to the objects with the smallest $r_{p}$ in our sample. A similar conclusion is reached by Casasola et al. (2004) with several hundred galaxies in pairs with $\mathrm{CO}$ data from the literature. They found that galaxies in pairs have more molecular gas than normal galaxies; however, the gas does not seem to be more efficient in forming stars.

Some studies propose opposite results, favoring SFE as the prime driver of interaction-induced star formation. Solomon \& Sage (1988) found that only strong interactions show enhanced SFE. Sofue et al. (1993) found an elevated SFE in Arp peculiar galaxies. The fact that SFE is enhanced in our close pairs is in agreement with these studies, since these are strongly interacting galaxies and Arp peculiar galaxies that, by definition, must be close to each other. However, these studies find no difference in total gas mass between isolated galaxies and galaxies in pairs, which leads to the conclusion that SFE is the determining factor in triggering star formation. It is not clear where this discrepancy stems from. The possible sources of the discrepancy could be the choice of $\alpha_{\mathrm{CO}}$ and the definition of the isolated (control) galaxy sample. In fact, many widely separated spectroscopic pairs show no obvious distortion in their morphology but do show enhancement of SFR, $M_{\mathrm{H}_{2}}$, and $f_{\text {gas. }}$. This emphasizes the importance of large spectroscopic data sets for identifying pairs and controls.

The physical origin of the enhanced $M_{\mathrm{H}_{2}}$ and $f_{\mathrm{gas}}$ is still unclear. One possible reason for the enhancement is an efficient transition from atomic to molecular gas by external pressure, as suggested by Kaneko et al. (2017). The cause of the external pressure can be the widespread shocks produced by interaction prevailing throughout a galaxy and cloud-cloud collisions in colliding regions (Icke 1985; Barnes 2004; Braine et al. 2004; Roche et al. 2015). Such an acceleration of the H I-to- $\mathrm{H}_{2}$ transition occurs even in the early stages of interaction (Kaneko et al. 2017). Moreover, Braine \& Combes (1993) proposed a scenario in which the gravitational torque induced by galaxy interaction provokes the infall of diffuse ionized halo gas inward. The ionized gas progressively turns into atomic gas because cooling becomes more efficient with increasing density. Merger simulations by Moreno et al. (2018) indeed find that ionized gas is depleted during the interaction phase 
probed by galaxy pairs. At a certain radius, because of the high density and cold temperature, the gas enters the molecular phase, causing growth of the $\mathrm{H}_{2}$ mass. The scenario is supported by cosmological hydrodynamical simulations (Moster et al. 2011) and binary merger simulations (Moreno et al. 2018). Moreover, the origin of the enhanced $M_{\mathrm{H}_{2}}$ and $f_{\text {gas }}$ might be somewhat analogous to what is being encountered by galaxies in clusters. Mok et al. $(2016,2017)$ showed that Virgo cluster galaxies have a significantly higher $\mathrm{H}_{2}$-to-H I ratio than the group sample. They interpret it as being a result of the various forms of interaction between galaxies that lead to gas flowing toward the center of host galaxies and the creation of $\mathrm{H}_{2}$. However, Ellison et al. $(2015,2018)$ showed that there is no decrease in $\mathrm{H}$ I in latestage galaxy mergers. It could still be possible that the $\mathrm{HI}$ reservoir fuels the $\mathrm{H}_{2}$ but that the interplay with other phases also plays a role and replenishes the H I (e.g., Moreno et al. 2018).

The impact of galaxy interactions is less evident for SFE (except for the closest and equal-mass pairs) when considering the globally averaged properties. The comparable integrated SFE of local early-stage pairs and isolated systems is in line with theoretical predictions. The simulations of Renaud et al. (2014) found that, on a galaxy-wide scale, approaching pairs are forming stars with similar efficiency as local spirals because the gravitational interaction and inflow are too weak to significantly increase the gas density. As many of our galaxies in pairs are in an early-stage interaction with their companion (i.e., two separated galaxies), the normal SFE of our galaxies in pairs is not surprising. Besides, we cannot exclude the possibility that galaxy interactions affect SFE at a much smaller scale than $f_{\text {gas }}$, such as the nuclear region, collision front, or particular side (e.g., leading or trailing) with respect to the interaction, and thus the variation in SFE is averaged out in a galaxy-wide study. The small-scale variation in SFE may also contribute to the large scatter of SFE among the galaxies in pairs (Figure 8), as the measured global SFE would depend on the observed area of a galaxy and various projection effects.

As the merger proceeds, the nuclear gas surface density of gas gets boosted by gravitational torques and inflows, making the gas more efficient at converting gas into stars (Mihos \& Hernquist 1996; Renaud et al. 2014; Sparre \& Springel 2016; Bustamante et al. 2018), and the system moves to its starburst phase. In agreement with the simulations, the SFE appears to be enhanced in our close pairs undergoing a strong interaction. However, it is worth cautioning that while nuclear starbursts are frequent among mergers (in both simulations and observations), observationally, there is a significant fraction of systems in which interaction-triggered star formation is taking place outside the nuclear region (García-Marín et al. 2009; CortijoFerrero et al. 2017; H.-A. Pan et al. 2018, in preparation; Thorp et al. 2019). Simulations based on the standard star formation model, i.e., in which the local SFR is related to the local gas density, often fail to reproduce this large-scale star formation (e.g., Barnes 2004; Chien \& Barnes 2010; Bournaud et al. 2011) and underestimate the SFR in regions where gas exhibits large velocity dispersions (e.g., Mihos et al. 1993; Barnes 2004; Chien \& Barnes 2010; Bournaud et al. 2011). Shockinduced and clustered star formation have been suggested to better account for the large-scale star formation in many interacting galaxies (e.g., Jog \& Solomon 1992; Barnes 2004; Saitoh et al. 2009; Teyssier et al. 2010; Powell et al. 2013).
Finally, we should note that, as pointed out by Violino et al. (2018), the decrease of SFR-matched $\Delta M_{\mathrm{H}_{2}}$ and $\Delta f_{\text {gas }}$ implies that internal mechanisms in isolated galaxies can have an effect similar to that caused by galaxy interactions. Mechanisms such as a bar instability could be a potential driver to accelerate atomic-to-molecular gas transitions (Masters et al. 2012) and promote star formation (Martinet \& Friedli 1997; Ellison et al. 2011; Carles et al. 2016; Kim et al. 2017) in isolated galaxies. However, the formation of bars may be closely tied with galaxy interactions (Noguchi 1987; Lang et al. 2014; Łokas et al. 2016).

\section{3. $S F R-M_{\mathrm{H}_{2}}$ Relation}

The SFR (or SFR surface density) is observed to correlate with $M_{\mathrm{H}_{2}}$ (or $M_{\mathrm{H}_{2}}$ surface density) with a power-law index of $N \approx 1-2$ (Kennicutt \& Evans 2012). Figure 11 illustrates the integrated $\mathrm{SFR}-M_{\mathrm{H}_{2}}$ relation of our galaxies in pairs (red circles) and controls (gray squares). The two populations largely overlap on the SFR $-M_{\mathrm{H}_{2}}$ plane, as also observed for the nearby galaxies in pairs in Violino et al. (2018; see their Figure 8). This is a consequence of the similar SFE between galaxies in pairs and controls (at all but the smallest separations). The linear least-squares fits $\left(\log (\mathrm{SFR})=N \times \log M_{\mathrm{H}_{2}}+b\right)$ yield a slope of $\sim N \approx 1.11 \pm 0.15$ for both galaxies in pairs and controls.

In order to better understand the role of galaxy interactions on star formation and connect the different galaxy populations, we compare our galaxies with 23 local isolated normal galaxies (green diamonds), 110 local galaxies in the Virgo cluster and nearby clouds (yellow pentagons), 19 local isolated (U)LIRGs (blue triangles), 49 local (U)LIRG mergers (orange hexagons), and 26 high- $z$ (U)LIRGs (purple thin diamonds).

Local isolated normal galaxies and (U)LIRGs. The local isolated normal galaxies and (U)LIRGs are taken from Gao \& Solomon (2004). An infrared luminosity of $10^{11} L_{\odot}$ is used to distinguish between normal galaxies and (U)LIRGs. Only galaxies that were observed in ${ }^{12} \mathrm{CO}(1-0)$ with the IRAM $30 \mathrm{~m}$ and NRAO $12 \mathrm{~m}$ telescopes are used in this work. Galaxies in Gao \& Solomon (2004) that have been classified as galaxy pairs and group galaxies from the NASA/IPAC Extragalactic Database $(\mathrm{NED})^{34}$ were removed (see Table 1 in Gao \& Solomon 2004); however, we cannot completely rule out the possibility of these (U)LIRGs being mergers. Most of the normal isolated objects are NGC galaxies, while the isolated (U)LIRGs are IRAS and Markarian galaxies.

Local galaxies in the Virgo cluster and nearby clouds. The local galaxies in dense environments, including the Virgo cluster and nearby clouds, are taken from the Herschel Reference Sample ${ }^{35}$ (HRS; Boselli et al. 2010). The nearby clouds are Leo, Ursa Major and Ursa Major Southern Spur, Crater, Coma I, Canes Venatici Spur and Canes VenaticiCamelopardalis, and Virgo-Libra Clouds. Galaxies are removed from the analysis if they have been identified as early type by NED or are located at the Virgo outskirts. The molecular gas data in ${ }^{12} \mathrm{CO}(1-0)$ for the HRS are either obtained at the Kitt Peak $12 \mathrm{~m}$ telescope or compiled from the literature (Boselli et al. 2014).

Local (U)LIRG mergers. The measurements of local (U) LIRG mergers are compiled from Gao \& Solomon (1999). The

\footnotetext{
34 https://ned.ipac.caltech.edu/

35 https://hedam.lam.fr/HRS/
} 
(a)

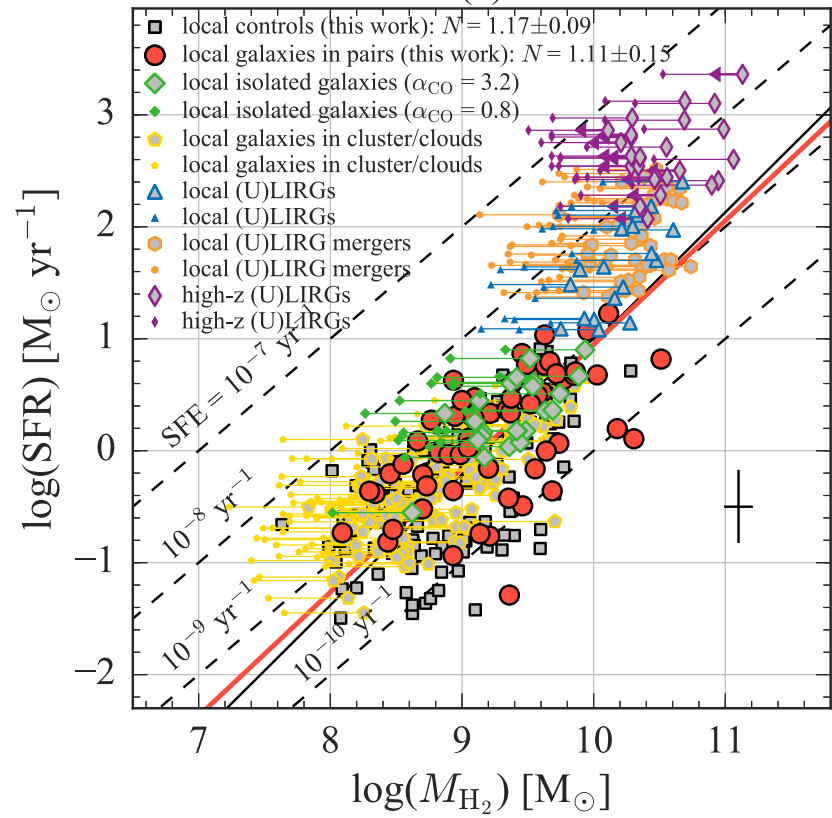

(b)

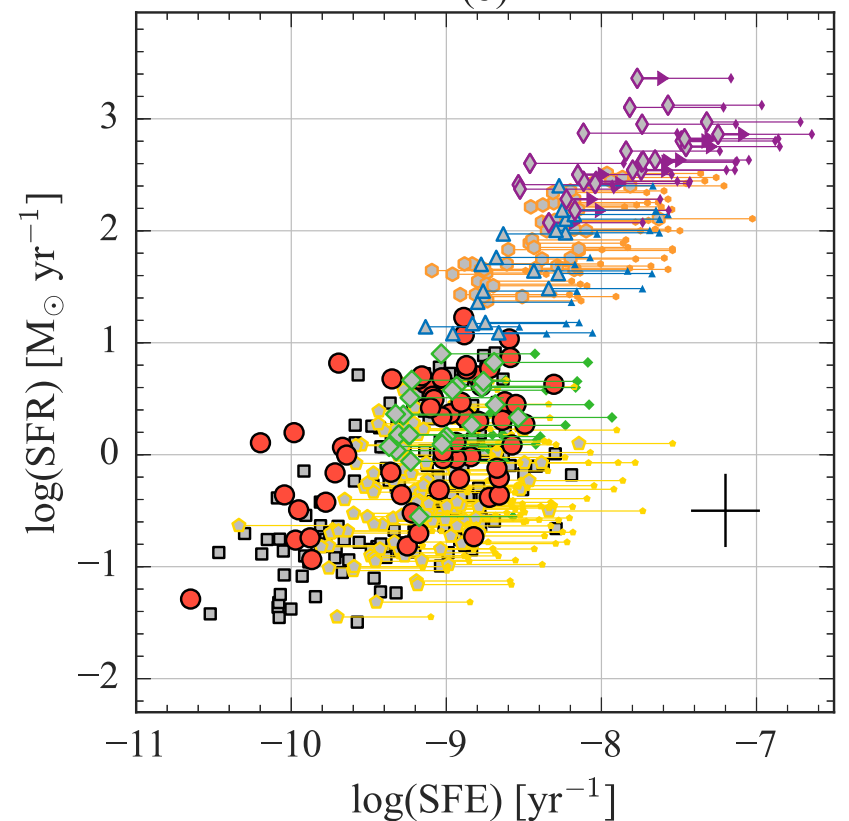

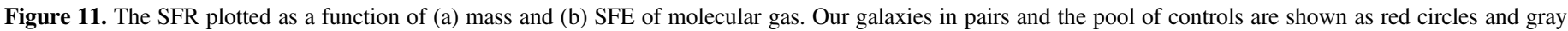

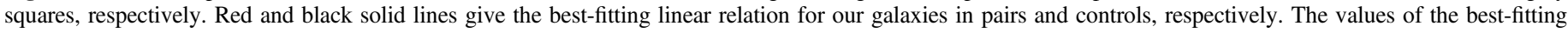

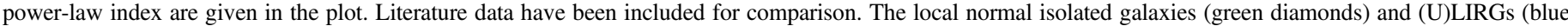

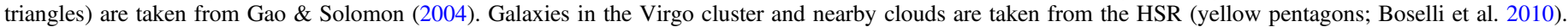

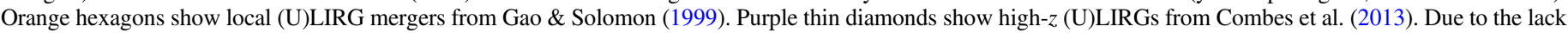

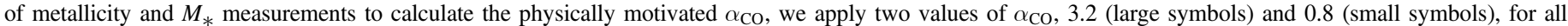

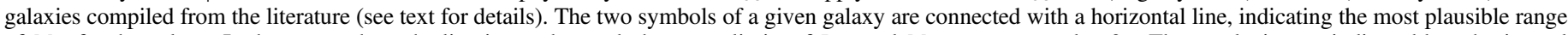

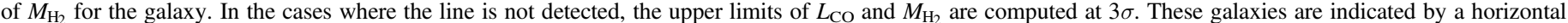

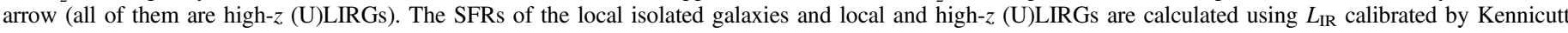

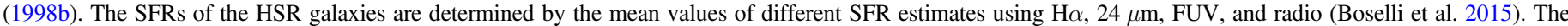

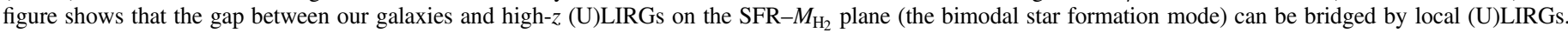
Moreover, the high SFR of high- $z$ (U)LIRGs is due to not only an enhancement of the molecular gas reservoir but also the SFE of the molecular gas.

observations were made with the IRAM $30 \mathrm{~m}$, NRAO $12 \mathrm{~m}$, and SEST $15 \mathrm{~m}$ telescopes at ${ }^{12} \mathrm{CO}(1-0)$. The (U)LIRG mergers are IRAS, Markarian, and Arp galaxies.

High-z $(U) L I R G$. The high $-z(0.2<z<1.0)$ (U)LIRGs are taken from Combes et al. (2013). Data of multiple CO transitions were taken, but in Figure 11, we only use the galaxies with ${ }^{12} \mathrm{CO}(2-1)$ observed with the IRAM $30 \mathrm{~m}$ telescope, which is the lowest transition in Combes et al. (2013). Combes et al. (2013) assumed a line ratio of 1 between the ${ }^{12} \mathrm{CO}(2-1)$ and ${ }^{12} \mathrm{CO}(1-0)$, as expected for warm, optically thick, and thermally excited gas in starburst objects. About half of these high- $z$ (U)LIRGs are interacting or merging systems, while the remaining objects appear unperturbed. Because of the increasing gas fraction with redshift, high- $z$ galaxies can easily be (U)LIRGs without violent interactions (e.g., Davé et al. 2010; Kartaltepe et al. 2012). Moreover, the high fraction of unperturbed high- $z$ (U)LIRGs is in part due to the low resolution and sensitivity when imaging high- $z$ galaxies. In those cases where the line is not detected, the upper limits of $L_{\mathrm{CO}}$ and $M_{\mathrm{H}_{2}}$ are computed at $3 \sigma$.

Due to the lack of metallicity and $M_{*}$ measurements necessary to calculate the physically motivated $\alpha_{\mathrm{CO}}$, we apply two values for $\alpha_{\mathrm{CO}}, 3.2$ and 0.8 , for all galaxies compiled from the literature. The higher $\alpha_{\mathrm{CO}}$ is the median value of our galaxies in pairs and controls; the lower $\alpha_{\mathrm{CO}}$ is the commonly adopted conversion factor for (U)LIRGs and distant galaxies (e.g., Solomon et al. 1997; Downes \& Solomon 1998; Daddi et al. 2010; Combes et al. 2013). Both results are plotted in
Figure 11 with large symbols for $\alpha_{\mathrm{CO}}=3.2$ and small symbols for $\alpha_{\mathrm{CO}}=0.8$. The two symbols of a given galaxy are connected with a horizontal line, indicating the most plausible range of $M_{\mathrm{H}_{2}}$ for the galaxy. The range of $M_{\mathrm{H}_{2}}$ of nondetected galaxies is also computed based on the upper limits of $L_{\mathrm{CO}}$. These galaxies are indicated by a horizontal arrow.

The SFRs of the local isolated galaxies and local and high- $z$ (U)LIRGs are calculated using infrared luminosity $\left(L_{\mathrm{IR}}\right.$; Kennicutt 1998b),

$$
\frac{\mathrm{SFR}}{\left(M_{\odot} \mathrm{yr}^{-1}\right)}=4.5 \times 10^{-44} \frac{L_{\mathrm{IR}}}{\left(\mathrm{erg} \mathrm{s}^{-1}\right)},
$$

assuming a Salpeter IMF. The SFRs of the HSR galaxies are determined by the mean values of different SFR estimates using $\mathrm{H} \alpha, 24 \mu \mathrm{m}, \mathrm{FUV}$, and radio, along with a Salpeter IMF (Boselli et al. 2015). It is necessary to multiply these Salpeter SFRs by 0.625 to transform from Salpeter IMF to Kroupa IMF.

With $\alpha_{\mathrm{CO}}=3.2$, the local isolated galaxies and galaxies in the Virgo cluster and nearby clouds populate the same regime in SFR- $M_{\mathrm{H}_{2}}$ space as our galaxies in isolation and in pairs. The SFE of local galaxies could increase to up to $\sim 10^{-8} \mathrm{yr}^{-1}$ if the lower conversion factor is used. The choice of the lower conversion factor for local galaxies might not be realistic (e.g., Sandstrom et al. 2013; Cormier et al. 2018), but it characterizes a potential upper limit of local SFE.

No matter which $\alpha_{\mathrm{CO}}$ is used, the SFE increases from local non-(U)LIRG galaxies, to local (U)LIRGs and (U)LIRG mergers, to high- $z$ (U)LIRGs. The change of SFE across the 
galaxy populations can be better seen in Figure 11(b), where we plot the SFR versus SFE of the gas. In other words, the high SFR of high-z (U)LIRGs is due to not only an enhancement of the molecular gas reservoir but also the enhanced SFE of the gas.

The different SFEs of high- $z$ and local star-forming galaxies have previously been considered as "bimodal" (Daddi et al. 2010; Genzel et al. 2010). In other words, local star-forming galaxies and high- $z$ starburst galaxies are two distinct populations. However, Figure 11 shows that the gap in the relation that extends from our local galaxies to the high- $z$ (U) LIRGs is filled by local (U)LIRGs, confirming the finding of Saintonge et al. (2011).

Some of our galaxies in pairs, both major and minor mergers, may become infrared-bright galaxies between the first passage and final coalescence during which star formation is dramatically boosted (e.g., Di Matteo et al. 2008; Kartaltepe et al. 2012; Renaud et al. 2014; in fact, a few of our galaxies in pairs have SFRs comparable to those of local (U)LIRGs, as seen in Figure 11). Carpineti et al. (2015) found that around $65 \%$ of local LIRGs are minor mergers (see also Ellison et al. 2013, and their Figure 10). However, minor mergers typically do not induce enough gas into the nuclear region to generate ULIRG-level luminosities. In the local universe, the majority of ULIRGs are triggered by almost equal-mass, gas-rich systems in advanced merger stages (Dasyra et al. 2006; Hwang et al. 2010; Carpineti et al. 2015).

\subsection{Caveats of This Work}

One caveat of the total gas mass determination is the fact that traditionally, the SDSS only probes the central $3^{\prime \prime}$. However, metallicity gradients have been observed in galaxies with a typical gradient of $\sim-0.05 \mathrm{dex} \mathrm{kpc}^{-1}$ (Pilyugin et al. 2014; Ho et al. 2015; Belfiore et al. 2017). The influence of using nuclear metallicity instead of globally averaged metallicity to calculate $\alpha_{\mathrm{CO}}$ is presumably stronger for isolated galaxies than galaxies in pairs due to interaction-induced radial mixing of gas, which flattens the metallicity profiles (Ellison et al. 2008; MichelDansac et al. 2008; Kewley et al. 2010; Scudder et al. 2012; Thorp et al. 2019).

Another caveat to the total gas mass determination is the assumption of ${ }^{12} \mathrm{CO}(2-1)$ to ${ }^{12} \mathrm{CO}(1-0)$ ratio $\left(R_{21}\right)$ for the sample observed in the $J=2 \rightarrow 1$ transition. In this work, we adopted an $R_{21}$ of 0.8 , which is an average value of the spatially resolved $R_{21}$ of nearby galaxies (Leroy et al. 2009) and corresponds to optically thick gas with an excitation temperature of $\sim 10 \mathrm{~K}$. However, $R_{21}$ varies from region to region in the Milky Way and nearby galaxies: $\sim 0.6-1.0$ in the spiral arms and galactic centers (star-forming molecular clouds) and $<0.6$ in the interarm regions (dormant molecular clouds; e.g., Leroy et al. 2009; Koda et al. 2012; Pan et al. 2015). If a higher value of $R_{21}$ is adopted $\left(R_{21} \approx 0.9-1\right)$, the $M_{\mathrm{H}_{2}}$, as well as the $\Delta M_{\mathrm{H}_{2}}$ and $\Delta f_{\text {gas }}$ with respect to the controls (which are observed at $J=1 \rightarrow 0$ ), would decrease. However, such a high value of $R_{21}$ is only observed in the nuclear regions; given that $R_{21}$ decreases with increasing galactocentric radius (Leroy et al. 2009), the true value of the globally averaged $R_{21}$ should be lower than that. It is also unlikely that our galaxies in pairs are dominated by dormant clouds, since they are actively forming stars; therefore, a globally averaged $R_{21}<0.6$ is not possible. Accordingly, we argue that our results should be only minimally affected by the assumption for $R_{21}$. Nonetheless, it is important to note that a variation of the
${ }^{12} \mathrm{CO}(3-2)$-to- ${ }^{12} \mathrm{CO}(1-0)$ ratio as a function of the merger sequence of (U)LIRGs has been reported (Leech et al. 2010; Michiyama et al. 2016).

\section{Summary}

We investigate the effect of galaxy interactions on global molecular gas properties by studying a sample of 58 galaxies in pairs (Section 2.1) and 154 control galaxies (Section 2.2). Molecular gas properties are determined from observations with the JCMT $15 \mathrm{~m}$, PMO $14 \mathrm{~m}$, and CSO $10 \mathrm{~m}$ telescopes and supplemented with data from the xCOLD GASS and JINGLE surveys at ${ }^{12} \mathrm{CO}(2-1)$ and ${ }^{12} \mathrm{CO}(1-0)$. The main conclusions are summarized below.

1. The median values of the SFR, $M_{\mathrm{H}_{2}}$, and $f_{\text {gas }}$ distributions of the full pairs sample are higher compared with those of the full control (nonmerger) sample. The differences between the control sample and galaxies in pairs are confirmed statistically by the K-S test. On the other hand, the SFE distribution of galaxies in pairs is statistically indistinguishable from that of the control sample (Section 3.1 and Figure 4).

2. We compute offsets in $M_{\mathrm{H}_{2}}, f_{\text {gas }}$, and SFR on a galaxy-bygalaxy basis by identifying controls that are matched in redshift, stellar mass, and effective size. All gas property offsets $\left(\Delta M_{\mathrm{H}_{2}}, \Delta f_{\text {gas }}\right.$, and $\left.\Delta \mathrm{SFE}\right)$ increase with $\Delta \mathrm{SFR}$, implying that both the available gas reservoir and the SFE of the gas are expected to influence SFR. However, the correlations are stronger for $\Delta M_{\mathrm{H}_{2}}$ and $\Delta f_{\text {gas }}$ than for $\triangle \mathrm{SFE}$ in terms of correlation coefficients (Section 3.2 and Figures 4 and 6).

3. The $\Delta \mathrm{SFR}, \Delta M_{\mathrm{H}_{2}}$, and $\Delta f_{\text {gas }}$ all increase with decreasing pair separation $\left(r_{p}\right)$ over the range from $\sim 70$ to $10 \mathrm{kpc}$. However, any SFE enhancement is only significant at the smallest pair separations $\left(r_{p}<20 \mathrm{kpc}\right.$; Section 3.3 and Figure 8).

4. The $\Delta$ SFR, $\Delta M_{\mathrm{H}_{2}}$, and $\Delta f_{\text {gas }}$ also exhibit a trend with stellar mass ratio of the two galaxies in a pair. Statistically, higher enhancements are found in pairs with smaller mass ratio. We find no apparent trend between the mass ratio and $\triangle \mathrm{SFE}$; it seems that statistically, SFE enhancements only occur in equal-mass pairs $(|\mu| \approx 0$; Section 3.4 and Figure 9).

5. If we additionally match the galaxies in pairs in SFR with controls, the gas mass and fraction are still enhanced in galaxies in pairs with respect to the controls, although by a smaller factor (Section 4.1 and Figure 4.1).

6. Our local galaxies in pairs and controls are largely overlapping on the $\mathrm{SFR}-M_{\mathrm{H}_{2}}$ relation as a result of their comparable SFE $\left(=\mathrm{SFR} / M_{\mathrm{H}_{2}}\right)$. The SFE of our galaxies is an order of magnitude lower than that in the high- $z$ (U) LIRGs. The gap between our galaxies and high- $z$ (U)LIRGs on the SFR- $-M_{\mathrm{H}_{2}}$ plane can be bridged by local (U)LIRGs. Moreover, the high SFR of high- $z$ (U)LIRGs is due to not only an enhancement of molecular gas reservoir but also the SFE of the gas (Section 4.3 and Figure 11).

All of the above, taken together, lead to the conclusion that galaxy interactions modify the total molecular gas mass, molecular gas mass fraction, and SFR of a galaxy, although the strength of the effect is dependent on merger properties.

Here we have only accounted for integrated properties. The next step of this work is to probe the spatially resolved star 
formation and molecular gas properties. A direct comparison of spatially resolved $\triangle \mathrm{SFR}$ and molecular gas properties will extend our understanding of the star formation process in galaxy pairs, e.g., where the enhanced $M_{\mathrm{H}_{2}}, f_{\text {gas }}$, and SFR are actually to be found. ALMA observations of MaNGA galaxies will be ideal for carrying out such an analysis.

We thank the anonymous referee for helpful suggestions that improved the paper. The work is supported by the Academia Sinica under the Career Development Award AS-CDA-107-M03 and the Ministry of Science \& Technology of Taiwan under the grant MOST 107-2119-M-001-024-. C.D.W. acknowledges support from the Natural Sciences and Engineering Research Council of Canada. Y.G. acknowledges the support of National Key R\&D Program of China No. 2017YFA0402704, NSFC No. 11420101002, and the CAS Key Research Program of Frontier Sciences. L.C.H. was supported by the National Key R\&D Program of China (2016YFA0400702) and the National Science Foundation of China (11473002, 11721303). E.B. acknowledges support from the UK Science and Technology Facilities Council [grant No. ST/M001008/1]. M.J.M. acknowledges the support of the National Science Centre, Poland, through POLONEZ grant 2015/19/P/ST9/04010; this project has received funding from the European Union's Horizon 2020 research and innovation program under Marie Skłodowska-Curie grant agreement No. 665778.

The authors wish to recognize and acknowledge the very significant cultural role and reverence that the summit of Maunakea has always had within the indigenous Hawaiian community. We are most fortunate to have the opportunity to conduct observations from this mountain.

We are grateful to the MPA-JHU group for access to their data products and catalogs. The Starlink software (Currie et al. 2014) is currently supported by the East Asian Observatory.

The James Clerk Maxwell Telescope is operated by the East Asian Observatory on behalf of the National Astronomical Observatory of Japan; the Academia Sinica Institute of Astronomy and Astrophysics; the Korea Astronomy and Space Science Institute; and the Operation, Maintenance and Upgrading Fund for Astronomical Telescopes and Facility Instruments, budgeted from the Ministry of Finance (MOF) of China and administrated by the Chinese Academy of Sciences (CAS), as well as the National Key R\&D Program of China (No. 2017YFA0402700). Additional funding support is provided by the Science and Technology Facilities Council of the United Kingdom and participating universities in the United Kingdom and Canada. This material is based upon work at the Caltech Submillimeter Observatory, which is operated by the California Institute of Technology.

This research has made use of data from the HRS project. The HRS is a Herschel Key Programme utilizing Guaranteed Time from the SPIRE instrument team, ESAC scientists, and a mission scientist. The HRS data were accessed through the Herschel Database in Marseille (HeDaM; http://hedam.lam.fr) operated by CeSAM and hosted by the Laboratoire d'Astrophysique de Marseille.

This research has made use of the NASA/IPAC Extragalactic Database (NED), which is operated by the Jet Propulsion Laboratory, California Institute of Technology, under contract with the National Aeronautics and Space Administration.
Funding for the Sloan Digital Sky Survey IV has been provided by the Alfred P. Sloan Foundation, the U.S. Department of Energy Office of Science, and the Participating Institutions. The SDSS acknowledges support and resources from the Center for High-Performance Computing at the University of Utah. The SDSS website is http://www.sdss.org.

The SDSS is managed by the Astrophysical Research Consortium for the Participating Institutions of the SDSS Collaboration, including the Brazilian Participation Group, the Carnegie Institution for Science, Carnegie Mellon University, the Chilean Participation Group, the French Participation Group, Harvard-Smithsonian Center for Astrophysics, Instituto de Astrofsica de Canarias, The Johns Hopkins University, Kavli Institute for the Physics and Mathematics of the Universe (IPMU)/University of Tokyo, the Korean Participation Group, Lawrence Berkeley National Laboratory, Leibniz Institut für Astrophysik Potsdam (AIP), Max-Planck-Institut für Astronomie (MPIA Heidelberg), Max-Planck-Institut für Astrophysik (MPA Garching), Max-Planck-Institut für Extraterrestrische Physik (MPE), the National Astronomical Observatories of China, New Mexico State University, New York University, the University of Notre Dame, the Observatório Nacional/MCTI, The Ohio State University, Pennsylvania State University, Shanghai Astronomical Observatory, the United Kingdom Participation Group, Universidad Nacional Autónoma de México, the University of Arizona, the University of Colorado Boulder, the University of Oxford, the University of Portsmouth, the University of Utah, the University of Virginia, the University of Washington, the University of Wisconsin, Vanderbilt University, and Yale University.

\section{ORCID iDs}

Hsi-An Pan (ib https://orcid.org/0000-0002-1370-6964 Lihwai Lin (10 https://orcid.org/0000-0001-7218-7407 Bau-Ching Hsieh (i) https://orcid.org/0000-0001-5615-4904 Yang Gao (ib https://orcid.org/0000-0002-6316-1632 Jorge Barrera-Ballesteros (i) https://orcid.org/0000-00032405-7258

Amélie Saintonge (ib https://orcid.org/0000-0003-4357-3450

Christine D. Wilson (iD https://orcid.org/0000-0001-5817-0991 Ho Seong Hwang (10) https://orcid.org/0000-0003-3428-7612 Luis C. Ho (10) https://orcid.org/0000-0001-6947-5846 Elias Brinks (iD https://orcid.org/0000-0002-7758-9699 Angus Mok (i) https://orcid.org/0000-0001-7413-7534 Aeree Chung (i) https://orcid.org/0000-0003-1440-8552 Harriet Parsons (iD https://orcid.org/0000-0002-6327-3423 Martin Bureau (1D https://orcid.org/0000-0003-4980-1012 Mark T. Sargent (iD https://orcid.org/0000-0003-1033-9684 Eun Jung Chung (iD https://orcid.org/0000-0003-0014-1527 Tie Liu (iD https://orcid.org/0000-0002-5286-2564

\section{References}

Accurso, G., Saintonge, A., Catinella, B., et al. 2017, MNRAS, 470, 4750 Arimoto, N., Sofue, Y., \& Tsujimoto, T. 1996, PASJ, 48, 275 Baldwin, J. A., Phillips, M. M., \& Terlevich, R. 1981, PASP, 93, 5 Barnes, J. E. 2004, MNRAS, 350, 798

Barnes, J. E., \& Hernquist, L. 1996, ApJ, 471, 115

Belfiore, F., Maiolino, R., Maraston, C., et al. 2016, MNRAS, 461, 3111 Belfiore, F., Maiolino, R., Tremonti, C., et al. 2017, MNRAS, 469, 151 Blanton, M. R., Bershady, M. A., Abolfathi, B., et al. 2017, AJ, 154, 28 Bolatto, A. D., Wolfire, M., \& Leroy, A. K. 2013, ARA\&A, 51, 207 
Borne, K. D., Bushouse, H., Colina, L., et al. 1999, Ap\&SS, 266, 137 Boselli, A., Cortese, L., Boquien, M., et al. 2014, A\&A, 564, A67 Boselli, A., Eales, S., Cortese, L., et al. 2010, PASP, 122, 261 Boselli, A., Fossati, M., Gavazzi, G., et al. 2015, A\&A, 579, A102 Bothwell, M. S., Kennicutt, R. C., \& Lee, J. C. 2009, MNRAS, 400, 154 Bothwell, M. S., Maiolino, R., Cicone, C., Peng, Y., \& Wagg, J. 2016, A\&A, 595, A48

Bournaud, F., Chapon, D., Teyssier, R., et al. 2011, ApJ, 730, 4

Braine, J., \& Combes, F. 1992, A\&A, 264, 433

Braine, J., \& Combes, F. 1993, A\&A, 269, 7

Braine, J., Lisenfeld, U., Duc, P.-A., et al. 2004, A\&A, 418, 419

Brinchmann, J., Charlot, S., White, S. D. M., et al. 2004, MNRAS, 351, 1151

Bruzual, G., \& Charlot, S. 2003, MNRAS, 344, 1000

Bundy, K., Bershady, M. A., Law, D. R., et al. 2015, ApJ, 798, 7

Bustamante, S., Sparre, M., Springel, V., \& Grand, R. J. J. 2018, MNRAS, 479, 3381

Cao, C., Xu, C. K., Domingue, D., et al. 2016, ApJS, 222, 16

Carles, C., Martel, H., Ellison, S. L., \& Kawata, D. 2016, MNRAS, 463, 1074

Carpineti, A., Kaviraj, S., Hyde, A. K., et al. 2015, A\&A, 577, A119

Casasola, V., Bettoni, D., \& Galletta, G. 2004, A\&A, 422, 941

Casoli, F., Boisse, P., Combes, F., \& Dupraz, C. 1991, A\&A, 249, 359

Chang, Y.-Y., van der Wel, A., da Cunha, E., \& Rix, H.-W. 2015, ApJS, 219, 8

Charlot, S., Kauffmann, G., Longhetti, M., et al. 2002, MNRAS, 330, 876

Charlot, S., \& Longhetti, M. 2001, MNRAS, 323, 887

Chien, L.-H., \& Barnes, J. E. 2010, MNRAS, 407, 43

Cid Fernandes, R., Pérez, E., García Benito, R., et al. 2013, A\&A, 557, A86

Combes, F., García-Burillo, S., Braine, J., et al. 2013, A\&A, 550, A41

Combes, F., Prugniel, P., Rampazzo, R., \& Sulentic, J. W. 1994, A\&A, 281, 725

Cormier, D., Bigiel, F., Jiménez-Donaire, M. J., et al. 2018, MNRAS, 475, 3909

Cortijo-Ferrero, C., González Delgado, R. M., Pérez, E., et al. 2017, MNRAS, 467, 3898

Cox, T. J., Jonsson, P., Somerville, R. S., Primack, J. R., \& Dekel, A. 2008 , MNRAS, 384, 386

Currie, M. J., Berry, D. S., Jenness, T., et al. 2014, in ASP Conf. Ser. 485, Astronomical Data Analysis Software and Systems XXIII, ed. N. Manset \& P. Forshay (San Francisco, CA: ASP), 391

Daddi, E., Elbaz, D., Walter, F., et al. 2010, ApJL, 714, L118

Darg, D. W., Kaviraj, S., Lintott, C. J., et al. 2010a, MNRAS, 401, 1552

Darg, D. W., Kaviraj, S., Lintott, C. J., et al. 2010b, MNRAS, 401, 1043

Dasyra, K. M., Tacconi, L. J., Davies, R. I., et al. 2006, ApJ, 638, 745

Davé, R., Finlator, K., Oppenheimer, B. D., et al. 2010, MNRAS, 404, 1355

Davies, L. J. M., Robotham, A. S. G., Driver, S. P., et al. 2015, MNRAS, 452,616

Di Matteo, P., Bournaud, F., Martig, M., et al. 2008, A\&A, 492, 31

Di Matteo, P., Combes, F., Melchior, A.-L., \& Semelin, B. 2007, A\&A, 468, 61

Donoso, E., Yan, L., Tsai, C., et al. 2012, ApJ, 748, 80

Downes, D., \& Solomon, P. M. 1998, ApJ, 507, 615

Ellison, S. L., Catinella, B., \& Cortese, L. 2018, MNRAS, 478, 3447

Ellison, S. L., Fertig, D., Rosenberg, J. L., et al. 2015, MNRAS, 448, 221

Ellison, S. L., Mendel, J. T., Patton, D. R., \& Scudder, J. M. 2013, MNRAS, 435, 3627

Ellison, S. L., Nair, P., Patton, D. R., et al. 2011, MNRAS, 416, 2182

Ellison, S. L., Patton, D. R., Simard, L., \& McConnachie, A. W. 2008, AJ, 135,1877

Gao, Y., \& Solomon, P. M. 1999, ApJL, 512, L99

Gao, Y., \& Solomon, P. M. 2004, ApJS, 152, 63

García-Marín, M., Colina, L., Arribas, S., \& Monreal-Ibero, A. 2009, A\&A, 505, 1319

Genzel, R., Tacconi, L. J., Gracia-Carpio, J., et al. 2010, MNRAS, 407, 2091

Gunn, J. E., Siegmund, W. A., Mannery, E. J., et al. 2006, AJ, 131, 2332

Ho, I.-T., Kudritzki, R.-P., Kewley, L. J., et al. 2015, MNRAS, 448, 2030

Hsieh, B. C., Lin, L., Lin, J. H., et al. 2017, ApJL, 851, L24

Hwang, H. S., Elbaz, D., Dickinson, M., et al. 2011, A\&A, 535, A60

Hwang, H. S., Elbaz, D., Lee, J. C., et al. 2010, A\&A, 522, A33

Icke, V. 1985, A\&A, 144, 115

Jiang, X.-J., Wang, Z., Gu, Q., Wang, J., \& Zhang, Z.-Y. 2015, ApJ, 799, 92

Jog, C. J., \& Solomon, P. M. 1992, ApJ, 387, 152

Kaneko, H., Kuno, N., Iono, D., et al. 2017, PASJ, 69, 66

Kartaltepe, J. S., Dickinson, M., Alexander, D. M., et al. 2012, ApJ, 757, 23

Kauffmann, G., Heckman, T. M., Tremonti, C., et al. 2003b, MNRAS, 346, 1055

Kauffmann, G., Heckman, T. M., White, S. D. M., et al. 2003a, MNRAS, 341, 33

Kennicutt, R. C., \& Evans, N. J. 2012, ARA\&A, 50, 531
Kennicutt, R. C., Jr. 1998a, ApJ, 498, 541

Kennicutt, R. C., Jr. 1998b, ARA\&A, 36, 189

Kewley, L. J., Dopita, M. A., Sutherland, R. S., Heisler, C. A., \& Trevena, J. 2001, ApJ, 556, 121

Kewley, L. J., Rupke, D., Zahid, H. J., Geller, M. J., \& Barton, E. J. 2010, ApJL, 721, L48

Kim, E., Hwang, H. S., Chung, H., et al. 2017, ApJ, 845, 93

Knapen, J. H., Cisternas, M., \& Querejeta, M. 2015, MNRAS, 454, 1742

Koda, J., Scoville, N., Hasegawa, T., et al. 2012, ApJ, 761, 41

Koyama, S., Koyama, Y., Yamashita, T., et al. 2017, ApJ, 847, 137

Kroupa, P. 2001, MNRAS, 322, 231

Lambas, D. G., Tissera, P. B., Alonso, M. S., \& Coldwell, G. 2003, MNRAS, 346, 1189

Lang, M., Holley-Bockelmann, K., \& Sinha, M. 2014, ApJL, 790, L33

Larson, R. B., \& Tinsley, B. M. 1978, ApJ, 219, 46

Lee, J. C., Hwang, H. S., \& Ko, J. 2013, ApJ, 774, 62

Leech, J., Isaak, K. G., Papadopoulos, P. P., Gao, Y., \& Davis, G. R. 2010 , MNRAS, 406, 1364

Leroy, A. K., Bolatto, A., Gordon, K., et al. 2011, ApJ, 737, 12

Leroy, A. K., Walter, F., Bigiel, F., et al. 2009, AJ, 137, 4670

Leroy, A. K., Walter, F., Brinks, E., et al. 2008, AJ, 136, 2782

Lin, L., Koo, D. C., Weiner, B. J., et al. 2007, ApJL, 660, L51

Lin, L., Koo, D. C., Willmer, C. N. A., et al. 2004, ApJL, 617, L9

Łokas, E. L., Ebrová, I., del Pino, A., et al. 2016, ApJ, 826, 227

Mannucci, F., Cresci, G., Maiolino, R., Marconi, A., \& Gnerucci, A. 2010, MNRAS, 408, 2115

Martig, M., \& Bournaud, F. 2008, MNRAS, 385, L38

Martinet, L., \& Friedli, D. 1997, A\&A, 323, 363

Masters, K. L., Nichol, R. C., Haynes, M. P., et al. 2012, MNRAS, 424, 2180

Mendel, J. T., Simard, L., Palmer, M., Ellison, S. L., \& Patton, D. R. 2014, ApJS, 210, 3

Michel-Dansac, L., Lambas, D. G., Alonso, M. S., \& Tissera, P. 2008, MNRAS, 386, L82

Michiyama, T., Iono, D., Nakanishi, K., et al. 2016, PASJ, 68, 96

Mihos, J. C., Bothun, G. D., \& Richstone, D. O. 1993, ApJ, 418, 82

Mihos, J. C., \& Hernquist, L. 1996, ApJ, 464, 641

Mok, A., Wilson, C. D., Golding, J., et al. 2016, MNRAS, 456, 4384

Mok, A., Wilson, C. D., Knapen, J. H., et al. 2017, MNRAS, 467, 4282

Moreno, J., Torrey, P., Ellison, S. L., et al. 2015, MNRAS, 448, 1107

Moreno, J., Torrey, P., Ellison, S. L., et al. 2018, MNRAS, submitted

Moster, B. P., Macciò, A. V., Somerville, R. S., Naab, T., \& Cox, T. J. 2011 , MNRAS, 415, 3750

Narayanan, D., Bothwell, M., \& Davé, R. 2012, MNRAS, 426, 1178

Noguchi, M. 1987, MNRAS, 228, 635

Pan, H.-A., Kuno, N., Koda, J., et al. 2015, ApJ, 815, 59

Park, C., \& Choi, Y.-Y. 2009, ApJ, 691, 1828

Patton, D. R., Pritchet, C. J., Carlberg, R. G., et al. 2002, ApJ, 565, 208

Patton, D. R., Torrey, P., Ellison, S. L., Mendel, J. T., \& Scudder, J. M. 2013 , MNRAS, 433, L59

Pettini, M., \& Pagel, B. E. J. 2004, MNRAS, 348, L59

Pilyugin, L. S., Grebel, E. K., \& Kniazev, A. Y. 2014, AJ, 147, 131

Powell, L. C., Bournaud, F., Chapon, D., \& Teyssier, R. 2013, MNRAS, 434, 1028

Renaud, F., Bournaud, F., Kraljic, K., \& Duc, P.-A. 2014, MNRAS, 442, L33

Roche, N., Humphrey, A., Gomes, J. M., et al. 2015, MNRAS, 453, 2349

Saintonge, A., Catinella, B., Tacconi, L. J., et al. 2017, ApJS, 233, 22

Saintonge, A., Kauffmann, G., Wang, J., et al. 2011, MNRAS, 415, 61

Saintonge, A., Tacconi, L. J., Fabello, S., et al. 2012, ApJ, 758, 73

Saintonge, A., Wilson, C. D., Xiao, T., et al. 2018, MNRAS, 481, 3497

Saitoh, T. R., Daisaka, H., Kokubo, E., et al. 2009, PASJ, 61, 481

Salim, S., Rich, R. M., Charlot, S., et al. 2007, ApJS, 173, 267

Sanders, D. B., Soifer, B. T., Elias, J. H., et al. 1988, ApJ, 325, 74

Sandstrom, K. M., Leroy, A. K., Walter, F., et al. 2013, ApJ, 777, 5

Sargent, M. T., Daddi, E., Béthermin, M., et al. 2014, ApJ, 793, 19

Scudder, J. M., Ellison, S. L., Momjian, E., et al. 2015, MNRAS, 449, 3719

Scudder, J. M., Ellison, S. L., Torrey, P., Patton, D. R., \& Mendel, J. T. 2012, MNRAS, 426, 549

Sofue, Y., Wakamatsu, K.-I., Taniguchi, Y., \& Nakai, N. 1993, PASJ, 45, 43

Solomon, P. M., Downes, D., Radford, S. J. E., \& Barrett, J. W. 1997, ApJ, 478, 144

Solomon, P. M., \& Sage, L. J. 1988, ApJ, 334, 613

Sparre, M., \& Springel, V. 2016, MNRAS, 462, 2418

Springel, V., \& Hernquist, L. 2005, ApJL, 622, L9 
Taylor, E. N., Hopkins, A. M., Baldry, I. K., et al. 2011, MNRAS, 418, 1587 Teyssier, R., Chapon, D., \& Bournaud, F. 2010, ApJL, 720, L149

Thorp, M. D., Ellison, S. L., Simard, L., Sánchez, S. F., \& Antonio, B. 2019, MNRAS, doi: $10.1093 / \mathrm{mnrasl} / \mathrm{sly} 185$

Tremonti, C. A., Heckman, T. M., Kauffmann, G., et al. 2004, ApJ, 613, 898

Violino, G., Ellison, S. L., Sargent, M., et al. 2018, MNRAS, 476, 2591
Wake, D. A., Bundy, K., Diamond-Stanic, A. M., et al. 2017, AJ, 154, 86 Whitaker, K. E., van Dokkum, P. G., Brammer, G., \& Franx, M. 2012, ApJL, 754, L29

Wong, K. C., Blanton, M. R., Burles, S. M., et al. 2011, ApJ, 728, 119

Xu, C. K., Domingue, D., Cheng, Y.-W., et al. 2010, ApJ, 713, 330

Xu, C. K., Shupe, D. L., Béthermin, M., et al. 2012, ApJ, 760, 72 